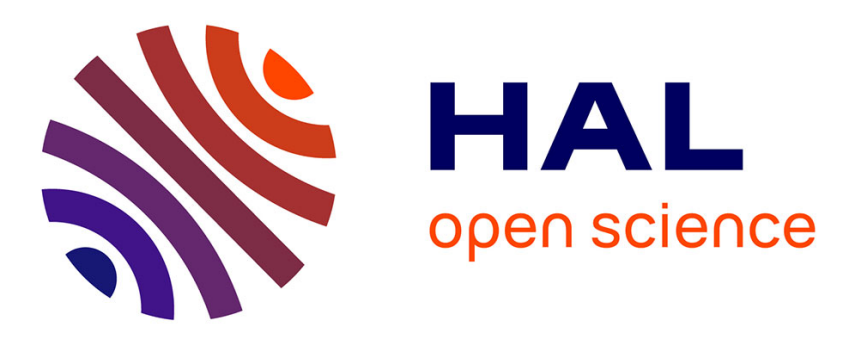

\title{
Eulerian model of immersed elastic surfaces with full membrane elasticity
}

Thomas Milcent, Emmanuel Maitre

\section{To cite this version:}

Thomas Milcent, Emmanuel Maitre. Eulerian model of immersed elastic surfaces with full membrane elasticity. Communications in Mathematical Sciences, 2016, 14 (3), pp.857-881. 10.4310/CMS.2016.v14.n3.a11 . hal-02047155v2

\section{HAL Id: hal-02047155 \\ https://hal.science/hal-02047155v2}

Submitted on 8 Oct 2019

HAL is a multi-disciplinary open access archive for the deposit and dissemination of scientific research documents, whether they are published or not. The documents may come from teaching and research institutions in France or abroad, or from public or private research centers.
L'archive ouverte pluridisciplinaire HAL, est destinée au dépôt et à la diffusion de documents scientifiques de niveau recherche, publiés ou non, émanant des établissements d'enseignement et de recherche français ou étrangers, des laboratoires publics ou privés. 


\title{
EULERIAN MODEL OF IMMERSED ELASTIC SURFACES WITH FULL MEMBRANE ELASTICITY*
}

\author{
THOMAS MILCENT ${ }^{\dagger}$ AND EMMANUEL MAITRE ${ }^{\ddagger}$
}

\begin{abstract}
We introduce an Eulerian model for the coupling of a fluid governed by the NavierStokes equations, with an immersed interface endowed with full membrane elasticity (i.e., including shear effects). We show numerical evidences of its ability to account for large displacements/shear in a relatively simple way, avoiding some drawbacks of Lagrangian representation.
\end{abstract}

Key words. Level set, fluid structure, Eulerian, immersed interface, Navier-Stokes, membrane elasticity.

AMS subject classifications. 74F10, 76D05, 65M06.

\section{Introduction}

In biomechanics of immersed membranes, the ability for a numerical model and method to handle shear efficiently is a challenging issue. Human red blood cells (RBCs) are among the simplest animal cells, since they have no nucleus nor organelle. They are made, like vesicles, of a phospholipid bilayer, plus a protein network (of spectrin), the cytoskeleton. For simple vesicles, the involved energy is a curvature energy, which is minimized with an area and enclosed volume constraint. For that case, Eulerian and Lagrangian models were developed in the last decade and proved to successfully reproduce the dynamics of such objects in flow $[5-9,11]$. For the red blood cells, the cytoskeleton provides extra resistance to membrane shear. Capsules are usually constituted of a liquid drop protected by a thin elastic membrane. They are used for applications in cosmetic, food, and pharmaceutical industries. They are more rigid objects than vesicles, so the curvature energy is small compared to membrane elasticity, including shear. In the modeling of vesicles or capsules, taking into account shear efficiently is of paramount interest.

On one hand, the definition of local shear variation on a surface is apparently easy in Lagrangian coordinates and, in the context of capsules, was formalized decades ago $[2,3,25]$. However, when it comes to practical computations, the unavoidable singularity of Lagrangian parametrization of closed surfaces brings high complications $[27,28]$. Moreover, large deformation and volume conservation in this Lagrangian setting also raise numerous difficulties, which were studied by several authors in the framework of the immersed-boundary method $[17,21]$. On the other hand, interface-capturing methods using level-set, while more intrinsic and dimension-independent regarding surface localization, are usually reported as unable to capture tangential motions (see, however, some connected studies on surface flows and multicomponent vesicles $[12,18,29]$ ). This is due to the fact that the transport equation for the level-set function, namely $\partial_{t} \phi+u \cdot \nabla \phi=0$, while recording some information on the surface area variations (enabling a complex fluid formulation of fluid-interface coupling $[4,8,9])$, just ignore any tangential component of $u$, since $\nabla \phi$ is normal to the interface.

\footnotetext{
*Received: October 31, 2014; accepted (in revised form): April 2, 2015. Communicated by John Lowengrub.

${ }^{\dagger}$ Institut de Mécanique de Bordeaux (I2M), Université de Bordeaux and Arts et Métiers ParisTech, France (thomas.milcent@u-bordeaux.fr).

${ }^{\ddagger}$ Laboratoire Jean Kuntzmann, Université de Grenoble-Alpes and CNRS, France (emmanuel. maitre@imag.fr).
} 
However, full 3D Eulerian elasticity and its coupling with fluid mechanics has already successfully been studied and implemented numerically by several teams both in the incompressible $[10,19,23,24,26,30]$ and compressible $[13,15]$ cases. The method relies on backward characteristics (also called a reference map by some authors) which map back material points to their initial positions. This concept was also introduced early for point correspondence in level-set applied to image processing [22] and more recently to improve numerical accuracy of advection equations (e.g. level-set motions) [16, 20].

This article is the first attempt, to the best of our knowledge, to write a full Eulerian model of an immersed interface endowed with full membrane elasticity (i.e. including shear effects) and to provide numerical evidences of its ability to account for large displacements/shear in a relatively simple way.

In Section 2, we recall the basic definitions of interface capturing via level-set and backward characteristics. In Section 3, we provide simple examples illustrating the possible drawbacks of a Lagrangian approach to record shear variations. In Section 4, we build two invariants that will be used to record the full membrane elasticity. The first one records area change (in both compressible and incompressible settings), while the second measures shear. In Section 5, we consider the fluid-structure coupling problem: an immersed membrane into an incompressible fluid. The whole model is reduced, following [10], to a Navier-Stokes equation with source terms expressed as a combination of partial derivatives of the backward characteristics, coupled with the vectorial transport equation giving these characteristics. We provide numerical illustrations of the ability of the numerical implementation of our model to simulate the dynamic relaxation of an initially sheared sphere. As the notion of shear is best understood through simple tests, we provide in Appendix A analytical examples of velocity fields under which the deformed continuous medium actually experiences a shear, and show that our invariants behave as expected. Appendix B deals with some technical lemmas.

\section{Preliminaries}

2.1. Forward and backward characteristics. Let $\Omega_{0} \subset \mathbb{R}^{3}$ be the reference configuration of a continuous medium and assume that this medium is deformed by a smooth map $X: \mathbb{R}^{3} \times \mathbb{R}^{+} \longrightarrow \mathbb{R}^{3}$ (the forward characteristics) to $\Omega_{t}=X\left(\Omega_{0}, t\right)$. A velocity field $u: \mathbb{R}^{3} \times \mathbb{R}^{+} \longrightarrow \mathbb{R}^{3}$ is naturally associated with $X$ :

$$
\partial_{t} X(\xi, t)=u(X(\xi, t), t), \quad X(\xi, 0)=\xi, \quad \xi \in \Omega_{0} .
$$

We introduce the backward characteristics $Y: \mathbb{R}^{3} \times \mathbb{R}^{+} \longrightarrow \mathbb{R}^{3}$ by the formula $Y(X(\xi, t), t)=\xi$ (see Figure 2.1). The physical interpretation of $Y(x, t)$ is the position at time 0 of a material particle lying in $x$ at time $t$ and moving at speed $u$. The derivative of this relation with respect to $t$ and $\xi$ in turn, gives with (2.1),

$$
\partial_{t} Y+\left(u \cdot \nabla_{x}\right) Y=0, \quad Y(x, 0)=x, \quad x \in \Omega_{t},
$$

and

$$
\left[\nabla_{\xi} X(\xi, t)\right]=\left[\nabla_{x} Y(x, t)\right]^{-1} \quad \text { for } x=X(\xi, t) .
$$

In the following, we use the notation $\nabla_{\xi}$ for the gradient with respect to $\xi$ and we denote by $\nabla$ the gradient with respect to $x$.

2.2. Eulerian representation of surfaces. We consider a surface $\Gamma_{t}$ captured by a level set function $\phi: \mathbb{R}^{3} \times \mathbb{R}^{+} \longrightarrow \mathbb{R}$

$$
\Gamma_{t}=\left\{x \in \mathbb{R}^{3} / \phi(x, t)=0\right\} .
$$


The initial position of the interface $\Gamma_{0}$ is associated to a given function $\phi_{0}$ (thus we set $\left.\phi(\cdot, 0)=\phi_{0}\right)$. We assume that the interface is advected by the velocity field $u$, such that $\phi$ verifies

$$
\partial_{t} \phi+u \cdot \nabla \phi=0
$$

We have, with (2.2), that the solution of (2.4) is given by

$$
\phi(x, t)=\phi_{0}(Y(x, t)) .
$$

Let the normal in the reference configuration be denoted by $n_{0}(\xi)$ for $\xi \in \Gamma_{0}$ and the normal in the deformed configuration by $n(x, t)$ for $x \in \Gamma_{t}$ (see Figure 2.1). These normals are expressed in terms of the normalized gradient of the associated level set:

$$
n(x, t)=\frac{\nabla \phi(x, t)}{|\nabla \phi(x, t)|}, \quad n_{0}(\xi)=\frac{\nabla_{\xi} \phi_{0}(\xi)}{\left|\nabla_{\xi} \phi_{0}(\xi)\right|} .
$$

The relation (2.5) gives $\nabla \phi(x, t)=[\nabla Y(x, t)]^{T} \nabla \phi_{0}(Y(x, t))$. Therefore, with the definitions (2.6),

$$
n_{0}(Y(x, t))=\frac{[\nabla Y(x, t)]^{-T} n(x, t)}{\left|[\nabla Y(x, t)]^{-T} n(x, t)\right|} .
$$

With (2.3), we get the Lagrangian equivalent of (2.7):

$$
n(X(\xi, t), t)=\frac{\left[\nabla_{\xi} X(\xi, t)\right]^{-T} n_{0}(\xi)}{\left|\left[\nabla_{\xi} X(\xi, t)\right]^{-T} n_{0}(\xi)\right|} .
$$

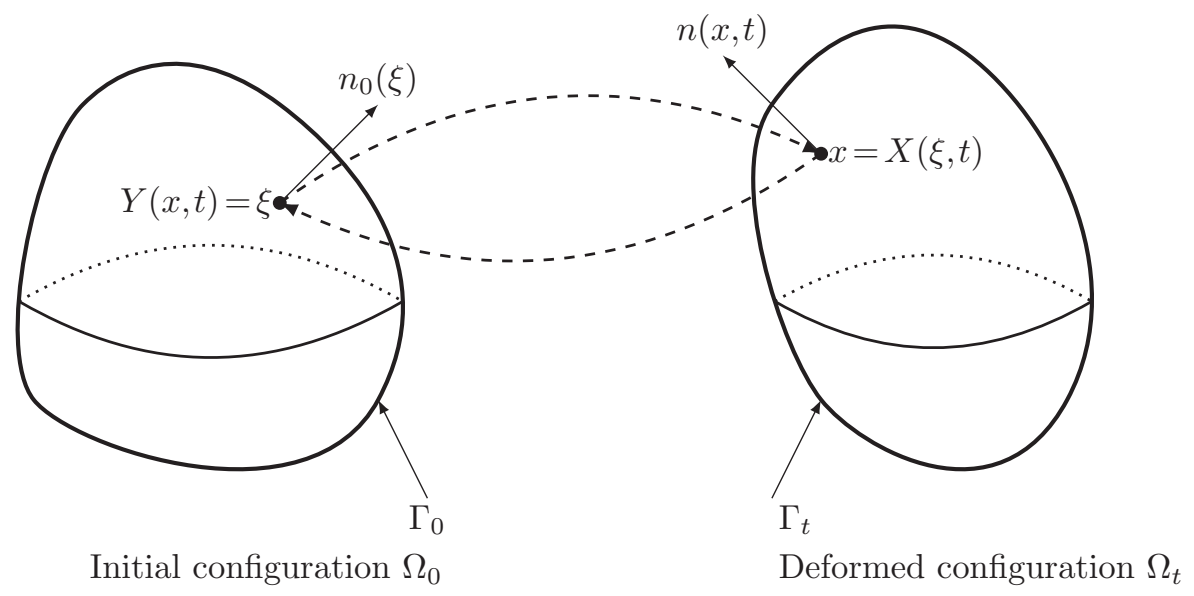

Fig. 2.1: Initial and deformed configurations.

\section{Can we measure surface shear variation with a Lagrangian description of the surface? \\ We present in this section two intuitive methods to measure shear variation with a parametrization of the surface. The first is based on the angle between two normalized vectors, and the second uses the invariants of the metric tensor. We will show that, even}


in the simplest case of a plane deformed in a shear velocity field, the two methods fail because the shear variation depends on the choice of initial parametrization. For general closed surfaces, more difficulties arise from the singularity of Lagrangian parametrizations. The first method cannot be applied because it is impossible to construct the normalized vectors (hairy ball theorem). We will show that, for a sphere deformed in a shear velocity field, the invariant of the second method is discontinuous at the poles. In any case, the definition of shear depends completely on the choice of the parametrization, and hence there is no canonical reference state. The limitations of these methods advocate for an immersion of the surface in $\mathbb{R}^{3}$ using a Eulerian description to capture shear.

3.1. Two Lagrangian methods to capture shear. Let $\Gamma_{0}$ be the initial surface described by the parametrization $\Psi_{0}: U \longrightarrow \mathbb{R}^{3}$, where $U$ is an open subset of $\mathbb{R}^{2}$. A parametrization of the deformed surface $\Gamma_{t}$ is given by $\Psi: U \times \mathbb{R}^{+} \longrightarrow \mathbb{R}^{3}$, where $\Psi\left(\theta_{1}, \theta_{2}, t\right)=X\left(\Psi_{0}\left(\theta_{1}, \theta_{2}\right), t\right)$ and the deformed vectors associated to the parametrization are given by $a_{i}(t)=\partial_{\theta_{i}} \Psi\left(\theta_{1}, \theta_{2}, t\right)$. The first intuitive method to capture shear is to consider the angle between the two normalized deformed vectors

$$
\operatorname{angle}(t)=\arccos \left(\frac{a_{1}(t)}{\left|a_{1}(t)\right|} \cdot \frac{a_{2}(t)}{\left|a_{2}(t)\right|}\right) .
$$

The second is to consider the metric tensor:

$$
\mathcal{M}(t):=\left(\begin{array}{ll}
a_{1}(t) \cdot a_{1}(t) & a_{1}(t) \cdot a_{2}(t) \\
a_{1}(t) \cdot a_{2}(t) & a_{2}(t) \cdot a_{2}(t)
\end{array}\right)
$$

which is classically used to compute area, angles, and length of curves on the surface. The trace of $\mathcal{M}(t)$ captures shear deformation but also contains information on local area. Dividing the trace by the square root of the determinant keeps only shear effects, as will be illustrated below. We thus introduce the following invariants:

$$
\mathcal{Z}_{1}(t)=\sqrt{\operatorname{det}(\mathcal{M}(t))}, \quad \mathcal{Z}_{2}(t)=\frac{\operatorname{Tr}(\mathcal{M}(t))}{2 \sqrt{\operatorname{det}(\mathcal{M}(t))}}
$$

where $\mathcal{Z}_{1}(t)$ correspond to the local area, and we will show that $\mathcal{Z}_{2}(t)$ is a good Lagrangian candidate to capture the local shear. In the following, we are interested in the local variation of area and shear between time 0 and $t$. Therefore, we consider the quantity angle $(t) / \operatorname{angle}(0)$ and the invariants $\mathcal{Z}_{i}(t) / \mathcal{Z}_{i}(0)$. In the following examples we consider the shear velocity field (see Figure A.10) with the associated forward characteristics given by

$$
u(x, y, z, t)=\left(\begin{array}{c}
0 \\
x \\
0
\end{array}\right), \quad X\left(\xi_{1}, \xi_{2}, \xi_{3}, t\right)=\left(\begin{array}{c}
\xi_{1} \\
\xi_{2}+t \xi_{1} \\
\xi_{3}
\end{array}\right) .
$$

3.2. Case of the plane: orthogonal parametrization. Let $\Gamma_{0}$ be the plane $\left\{(x, y, z) \in \mathbb{R}^{3} / z=0\right\}$ which is parametrized by $\Psi_{0}: \mathbb{R}^{2} \longrightarrow \mathbb{R}^{3}$ given by

$$
\Psi_{0}:\left(\theta_{1}, \theta_{2}\right) \mapsto\left(\begin{array}{c}
\cos (\alpha) \theta_{1}-\sin (\alpha) \theta_{2} \\
\sin (\alpha) \theta_{1}+\cos (\alpha) \theta_{2} \\
0
\end{array}\right) .
$$


where $\alpha \in[0,2 \pi]$ is a parameter. The deformed surface $\Gamma_{t}$ is still geometrically $\Gamma_{0}$ in this case, and the deformed vectors are given by

$$
a_{1}(t)=\left(\begin{array}{c}
\cos (\alpha) \\
\sin (\alpha)+t \cos (\alpha) \\
0
\end{array}\right), \quad a_{2}(t)=\left(\begin{array}{c}
-\sin (\alpha) \\
\cos (\alpha)-t \sin (\alpha) \\
0
\end{array}\right) .
$$

At $t=0$, these vectors are orthonormal and have an $\alpha$ angle with the canonical basis (see Figure 3.1). We can show that, for this example, we have

$$
\frac{\operatorname{angle}(t)}{\operatorname{angle}(0)}=\frac{2}{\pi} \arccos \left(\frac{t(2 \cos (2 \alpha)-t \sin (2 \alpha))}{2 \sqrt{\left(1+t \sin (2 \alpha)+t^{2} \cos (\alpha)^{2}\right)\left(1-t \sin (2 \alpha)+t^{2} \sin (\alpha)^{2}\right)}}\right) .
$$

This quantity depends on the angle parameter $\alpha$ (even if all pair of initial vectors are orthonormal). Hence, this first method is not appropriate to measure the local shear variation. For the second method, the invariants (3.1) in the case of the plane are given by

$$
\frac{\mathcal{Z}_{1}(t)}{\mathcal{Z}_{1}(0)}=1, \quad \frac{\mathcal{Z}_{2}(t)}{\mathcal{Z}_{2}(0)}=1+\frac{t^{2}}{2}
$$

Hence, there is no area variation and the shear is constant in space and increases with time, which correspond intuitively to what we can expect of a shear deformation. The second invariant is independent of $\alpha$, so this second method seems appropriate to capture shear.
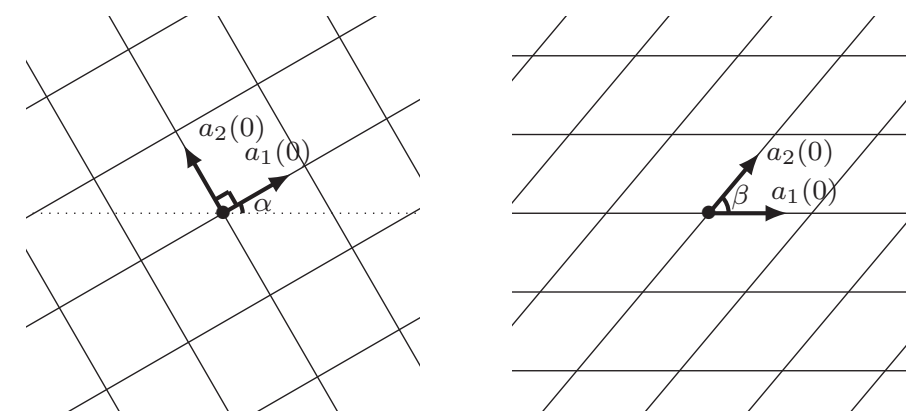

Fig. 3.1: Orthogonal (left) and non orthogonal (right) parametrization of the plane.

3.3. Case of the plane: non orthogonal parametrization. Let $\Gamma_{0}$ be the same plane $\left\{(x, y, z) \in \mathbb{R}^{3} / z=0\right\}$ described with another parametrization $\Psi_{0}: \mathbb{R}^{2} \longrightarrow \mathbb{R}^{3}$ given by

$$
\Psi_{0}:\left(\theta_{1}, \theta_{2}\right) \mapsto\left(\begin{array}{c}
\theta_{1}+\cos (\beta) \theta_{2} \\
\sin (\beta) \theta_{2} \\
0
\end{array}\right)
$$

where $\beta \in[0,2 \pi]$ is a parameter. The deformed vectors are given by

$$
a_{1}(t)=\left(\begin{array}{l}
1 \\
t \\
0
\end{array}\right), \quad a_{2}(t)=\left(\begin{array}{c}
\cos (\beta) \\
\sin (\beta)+t \cos (\beta) \\
0
\end{array}\right)
$$


At $t=0$, these vectors are not orthogonal and have a $\beta$ angle (see Figure 3.1). For the second method, the invariants (3.1) are given by

$$
\frac{\mathcal{Z}_{1}(t)}{\mathcal{Z}_{1}(0)}=1, \quad \frac{\mathcal{Z}_{2}(t)}{\mathcal{Z}_{2}(0)}=\frac{1}{2}\left(1+t^{2}+\cos (\beta)^{2}+(t \cos (\beta)+\sin (\beta))^{2}\right) .
$$

There is no area variation, and the shear depends on the parameter $\beta$. Therefore, the second method fails to capture local shear variation because it depends on the choice of the parametrization.

3.4. Case of the sphere: orthogonal parametrization. Let $\Gamma_{0}$ be the sphere $\left\{(x, y, z) \in \mathbb{R}^{3} / x^{2}+y^{2}+z^{2}=1\right\}$ which is parametrized by $\Psi_{0}:[0, \pi] \times[0,2 \pi] \longrightarrow \mathbb{R}^{3}$ given by

$$
\Psi_{0}:\left(\theta_{1}, \theta_{2}\right) \mapsto\left(\begin{array}{c}
\sin \left(\theta_{1}\right) \cos \left(\theta_{2}\right) \\
\sin \left(\theta_{1}\right) \sin \left(\theta_{2}\right) \\
\cos \left(\theta_{1}\right)
\end{array}\right)
$$

The deformed vectors are given by

$$
\begin{aligned}
& a_{1}(t)=\left(\begin{array}{c}
\cos \left(\theta_{1}\right) \cos \left(\theta_{2}\right) \\
\cos \left(\theta_{1}\right) \sin \left(\theta_{2}\right)+\cos \left(\theta_{1}\right) \cos \left(\theta_{2}\right) t \\
-\sin \left(\theta_{1}\right)
\end{array}\right), \\
& a_{2}(t)=\left(\begin{array}{c}
-\sin \left(\theta_{1}\right) \sin \left(\theta_{2}\right) \\
\sin \left(\theta_{1}\right) \cos \left(\theta_{2}\right)-\sin \left(\theta_{1}\right) \sin \left(\theta_{2}\right) t \\
0
\end{array}\right) .
\end{aligned}
$$

At $t=0$, these vectors are orthogonal (see Figure 3.2). The first method with angle between vectors also fails in this more complex case. Indeed, it is impossible to construct a continuous tangent vector field on a sphere (hairy ball theorem). For the second method, the invariants (3.1) in the case of the sphere are given by

$$
\begin{aligned}
& \frac{\mathcal{Z}_{1}(t)}{\mathcal{Z}_{1}(0)}=\sqrt{1-t \sin \left(\theta_{1}\right)^{2} \sin \left(2 \theta_{2}\right)+t^{2} \sin \left(\theta_{1}\right)^{2} \sin \left(\theta_{2}\right)^{2}} \\
& \frac{\mathcal{Z}_{2}(t)}{\mathcal{Z}_{2}(0)}=\frac{1+\sin \left(\theta_{1}\right)^{2}+t \cos \left(2 \theta_{1}\right) \sin \left(2 \theta_{2}\right)+\frac{t^{2}}{2}\left(1+\cos \left(2 \theta_{1}\right) \cos \left(2 \theta_{2}\right)\right)}{\left(1+\sin \left(\theta_{1}\right)^{2}\right) \sqrt{1-t \sin \left(\theta_{1}\right)^{2} \sin \left(2 \theta_{2}\right)+t^{2} \sin \left(\theta_{1}\right)^{2} \sin \left(\theta_{2}\right)^{2}}} .
\end{aligned}
$$

The first invariant (3.5) is well defined and represents the local area variation. The limit at the poles $\left(\theta_{1}=0\right.$ or $\left.\theta_{1}=\pi\right)$ of the second invariant $(3.6)$ is $\cos \left(\theta_{2}\right)^{2}+\left(\sin \left(\theta_{2}\right)+\right.$ $\left.t \cos \left(\theta_{2}\right)\right)^{2}$ and depends of $\theta_{2}$. Therefore, there is a discontinuity of the second invariant at the poles. We can reparametrize periodically the surface, but the algorithms are more complex. Moreover, the results will always depend on the choice of the parametrization, even in zones where there is no singularity.

To tackle this problem, we propose immersing the surface in $\mathbb{R}^{3}$ and projecting on it the $3 \mathrm{D}$ deformations. In this case, we use the flat metric of $\mathbb{R}^{3}$ instead of the metric of the surface and thus avoid the singularities of parametrizations. Also, the immersion in $\mathbb{R}^{3}$ will allow us to get a reference state, which does not depend on the parametrization. Furthermore, we will introduce $Z_{i}$, the Eulerian equivalent of the Lagrangian invariants $\mathcal{Z}_{i}$. We will show that $Z_{1}$ is the classical and well-known local-area variation. We will demonstrate with a variety of analytical illustrations in Appendix A that $Z_{2}$ is a good candidate to record the local shear variation. 


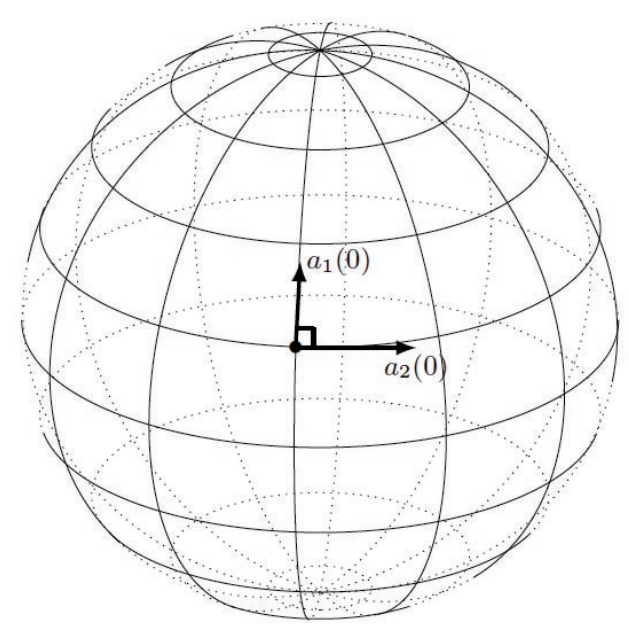

Fig. 3.2: Orthogonal parametrization of the sphere.

\section{Surface deformation in the Eulerian frame}

4.1. The surface tensor $\mathcal{A}$. We want to measure the deformations on the surface $\Gamma_{t}$. Following the Lagrangian description of $[2,3,25]$, we introduce the tensor

$$
M(X(\xi, t), t):=\left[\nabla_{\xi} X(\xi, t)\right]\left[\mathbb{I}-n_{0}(\xi) \otimes n_{0}(\xi)\right] .
$$

Let $v(\xi)$ be a vector defined at the point $\xi \in \Gamma_{0}$. This vector is first projected with $\left[\mathbb{I}-n_{0}(\xi) \otimes n_{0}(\xi)\right]$ to $v_{\tau}(\xi) \in T_{\xi} \Gamma_{0}$, the tangent plane of $\Gamma_{0}$ at $\xi$. Then the vector $v_{\tau}(\xi)$ is deformed with $X$ in the vector $\left[\nabla_{\xi} X(\xi, t)\right] v_{\tau}(\xi)$ at $X(\xi, t)$. A first property is that $M v$ is already in $T_{X(\xi, t)} \Gamma_{t}$, the tangent plane of $\Gamma_{t}$ at $X(\xi, t)$. Indeed, using (2.8) and $v_{\tau}(\xi) \cdot n_{0}(\xi)=0$, we have

$$
\left.(M(X(\xi, t), t) v(\xi)) \cdot n(X(\xi, t), t)=\left(\left[\nabla_{\xi} X\right]^{T} n(X(\xi, t), t)\right) \cdot v_{\tau}(\xi)\right)=0 .
$$

The tensor (4.1) is written in its Eulerian form with (2.3)

$$
M(x, t):=[\nabla Y(x, t)]^{-1}\left[\mathbb{I}-n_{0}(Y(x, t)) \otimes n_{0}(Y(x, t))\right] .
$$

The associate Cauchy-Green tensor is defined as follows $\left(\mathbb{I}-n_{0} \otimes n_{0}\right.$ is a projector hence involutive):

$$
\mathcal{A}:=M M^{T}=[\nabla Y]^{-1}\left(\mathbb{I}-n_{0}(Y) \otimes n_{0}(Y)\right)[\nabla Y]^{-T} .
$$

After introducing the right Cauchy-Green tensor

$$
B=\left[\nabla_{\xi} X\right]\left[\nabla_{\xi} X\right]^{T}=[\nabla Y]^{-1}[\nabla Y]^{-T},
$$

using (2.7), the relation $A(v \otimes v) A^{T}=(A v) \otimes(A v)$, and $\left|[\nabla Y]^{-T} n\right|^{2}=(B n) \cdot n$, we get

$$
\mathcal{A}=B-\frac{(B n) \otimes(B n)}{(B n) \cdot n} .
$$


4.2. Invariants of $\mathcal{A}$. According to (4.3), we have

$$
\mathcal{A} n=0 .
$$

Thus, 0 is an eigenvalue and $\operatorname{det}(\mathcal{A})=0$. The other invariants are $\operatorname{Tr}(\mathcal{A})$ and $\operatorname{Tr}(\operatorname{Cof}(\mathcal{A}))=\frac{1}{2}\left(\operatorname{Tr}(\mathcal{A})^{2}-\operatorname{Tr}\left(\mathcal{A}^{2}\right)\right)$. As $\mathcal{A}$ is real and symmetric, there exists an orthonormal basis of eigenvectors. Moreover, $\mathcal{A}$ is positive since $\mathcal{A} x \cdot x=\left|M^{T} x\right|^{2} \geq 0$, so we denote its eigenvalues by $0, \lambda_{1}^{2}$ and $\lambda_{2}^{2}$. Therefore, $\operatorname{Tr}(\mathcal{A})=\lambda_{1}^{2}+\lambda_{2}^{2}$ and $\operatorname{Tr}(\operatorname{Cof}(\mathcal{A}))=\left(\lambda_{1} \lambda_{2}\right)^{2}$. We introduce the following invariants:

$$
\begin{aligned}
& Z_{1}=\sqrt{\operatorname{Tr}(\operatorname{Cof}(\mathcal{A}))}=\left|\lambda_{1} \lambda_{2}\right|, \\
& Z_{2}=\frac{\operatorname{Tr}(\mathcal{A})}{2 \sqrt{\operatorname{Tr}(\operatorname{Cof}(\mathcal{A}))}}=\frac{1}{2}\left(\left|\frac{\lambda_{1}}{\lambda_{2}}\right|+\left|\frac{\lambda_{2}}{\lambda_{1}}\right|\right) .
\end{aligned}
$$

In the reference configuration (usually at initial time in our case), we have that $\mathcal{A}(0)=$ $\mathbb{I}-n_{0} \otimes n_{0}$ so $\operatorname{Tr}(\mathcal{A}(0))=2$ and $\operatorname{Tr}(\operatorname{Cof}(\mathcal{A}(0)))=1$. Under the smoothness assumption on $u$, there exists a time $T$ that for $t<T, \operatorname{Tr}(\mathcal{A})$ and $\operatorname{Tr}(\operatorname{Cof}(\mathcal{A}))$ do not vanish. Therefore, the invariant $Z_{2}$ is well defined. We have that $Z_{1}=Z_{2}=1$ at initial time and the inequalities $Z_{1} \geq 0, Z_{2} \geq 1$. Note that $Z_{1}$ and $Z_{2}$ are both Eulerian fields, and in 5.2 we will derive equations to compute them. We will prove in Appendix B that $Z_{1}$ is exactly equal to the local area variation of the surface (even if incompressibility is not assumed on $u$ ). We will demonstrate in Appendix $\mathrm{A}$ that $Z_{2}$ is a good candidate to record the local shear variation.

\section{Immersed membrane in a fluid}

In this section, we apply the above considerations to build an Eulerian fluidstructure model of an immersed elastic interface, which accounts for the full membrane elasticity through an energy involving invariants $Z_{1}$ and $Z_{2}$. We derive Eulerian equations verified by the two invariants, and the forces induced by the energy variation during the structure motion. The final model is recast as a Navier-Stokes system with a source term, the latter depending on space derivatives of $Z_{1}$ and $Z_{2}$, coupled with their Eulerian equations. This is a generalization to full membrane elasticity of a simple level-set formulation of the immersed boundary method that was introduced previously and that we recall now.

5.1. Mathematical model. We consider an elastic membrane $\Gamma_{t}$ immersed into a fluid governed by, for example incompressible Navier-Stokes equations, lying in a bounded domain $\Omega$ of $\mathbb{R}^{3}$, for a time interval $[0, T]$. The interface $\Gamma_{t}$ is captured by a level-set function $\phi: \Omega \times[0, T] \rightarrow \mathbb{R}$. Based on previous works on Eulerian description of immersed interface $[8,9]$, we write the coupling between the immersed surface and the surrounding fluid under the formulation

$$
\begin{cases}\partial_{t} u+u \cdot \nabla u-\frac{1}{R_{e}} \Delta u+\nabla p=f(\phi) & \text { on } \Omega \times[0, T] \\ \operatorname{div} u=0 & \text { on } \Omega \times[0, T] \\ \partial_{t} \phi+u \cdot \nabla \phi=0 & \text { on } \Omega \times[0, T]\end{cases}
$$

where $f(\phi)$ accounts for the elastic force imparted on fluid by the immersed surface. Dependence on $\phi$ means here dependence on $\phi$ and its derivatives. We have shown that, in the incompressible case, $|\nabla \phi|$ captures the local area variation and that the force associated to the regularized energy

$$
\mathcal{E}=\int_{\Omega} E(|\nabla \phi|) \frac{1}{\varepsilon} \zeta\left(\frac{\phi}{\varepsilon}\right) \mathrm{d} x
$$


can be expressed by the formula

$$
f(\phi)=\operatorname{div}\left[E^{\prime}(|\nabla \phi|)|\nabla \phi|\left(\mathbb{I}-\frac{\nabla \phi \otimes \nabla \phi}{|\nabla \phi|^{2}}\right) \frac{1}{\varepsilon} \zeta\left(\frac{\phi}{\varepsilon}\right)\right]
$$

where $r \mapsto E(r)$ is the elastic constitutive law, $\varepsilon$ is the width of the interface, and $\zeta$ is a cut-off function used to spread the interface near $\{\phi=0\}$. However, in order to capture the full membrane energy, the function $\phi$ is not sufficient. Indeed a velocity field $u$ tangential to $\Gamma_{t}$ would verify $u \cdot \nabla \phi=0$ and therefore would not change the value of $\phi$ in its evolution equation. In order to capture some information on how points are moving on the surface, one way is to use the backward characteristics $Y$ of the velocity field $u$. We propose the new model:

$$
\begin{cases}\partial_{t} u+u \cdot \nabla u-\frac{1}{R_{e}} \Delta u+\nabla p=F_{1}(\phi, Y)+F_{2}(\phi, Y) & \text { on } \Omega \times[0, T] \\ \operatorname{div} u=0 & \text { on } \Omega \times[0, T] \\ \partial_{t} Y+u \cdot \nabla Y=0 & \text { on } \Omega \times[0, T]\end{cases}
$$

where $F_{i}$ is the force associated with the invariant $Z_{i}$, which expressions will be derived in the next section. In most cases, equations (5.3) will be supplemented with homogeneous initial and boundary conditions on the velocity, and with initial condition on $Y$ given by the identity

$$
u=0 \text { on } \partial \Omega \times[0, T], \quad u=0 \text { on } \Omega \times\{0\}, \quad Y(0, x)=x \text { on } \Omega .
$$

Under such initial and boundary conditions, characteristics remain for all time inside $\Omega$. Thus the initial and deformed whole continuous medium (fluid and structure) always occupies the same domain of $\mathbb{R}^{3}$ (thus there is no need to introduce an initial and deformed configuration as in 2.1).

Moreover, with such initial and boundary conditions, there is no need to solve an equation on $\phi$, since the knowledge of $Y$ and $\phi_{0}$ is sufficient. Indeed, the solution to the transport equation on $\phi$ is given by the following (see $(2.5)$ ):

$$
\phi(x, t)=\phi_{0}(Y(x, t)) .
$$

However if we consider an initially deformed interface (see (5.20) in the numerical tests), we could choose to set $Y(0, \cdot)$ to some maps not equal to the identity. In that case, we need to solve the transport equation on $\phi$

$$
\partial_{t} \phi+u \cdot \nabla \phi=0
$$

REMARK 5.1. One could wonder about the optimality of our representation, regarding the number of fields to capture full membrane deformation. Note that, to capture the interface position itself, we need one field (a level-set function $\phi$ ). In the general case, to record the area change, we need to compute $Z_{1}$ (or $|\nabla \phi|$ and $J$; see (B.1) in Appendix B), and therefore an extra field to get the shear. Thus, as we solve for $Y$, which has three components, and obtain $\phi, Z_{1}$ and $Z_{2}$ from it, we are optimal. In the incompressible case, area change is recorded by $|\nabla \phi|$ alone. We are not able, for the moment, to express shear by only introducing another field that we could compute without involving $Y$. 
5.2. Eulerian equations on $Z_{i}$. In order to build an Eulerian model, we need to find evolution equations associated with Eulerian invariants.

Proposition 5.1. Under the smoothness assumption made on $u$, the invariants verify

$$
\begin{array}{ll}
\partial_{t} Z_{1}+u \cdot \nabla Z_{1}=Z_{1}[\nabla u]: \mathcal{C}_{1}, & \mathcal{C}_{1}=\mathbb{I}-n \otimes n, \\
\partial_{t} Z_{2}+u \cdot \nabla Z_{2}=Z_{2}[\nabla u]: \mathcal{C}_{2}, & \mathcal{C}_{2}=\frac{2 \mathcal{A}}{\operatorname{Tr}(\mathcal{A})}-(\mathbb{I}-n \otimes n) .
\end{array}
$$

Proof. We first focus on the equations related to $\mathcal{A}$. Taking the gradient of (2.2), we get

$$
\partial_{t}[\nabla Y]+u \cdot \nabla([\nabla Y])=-[\nabla Y][\nabla u]
$$

and its inverse verifies

$$
\partial_{t}\left([\nabla Y]^{-1}\right)+u \cdot \nabla\left([\nabla Y]^{-1}\right)=[\nabla u][\nabla Y]^{-1} .
$$

The equation (4.2) on $B$ is then given by

$$
\partial_{t} B+u \cdot \nabla B=[\nabla u] B+B[\nabla u]^{T} .
$$

Using (2.2) we get that

$$
\partial_{t}\left(\mathbb{I}-n_{0}(Y) \otimes n_{0}(Y)\right)+u \cdot \nabla\left(\mathbb{I}-n_{0}(Y) \otimes n_{0}(Y)\right)=0 .
$$

This relation together with (5.7) gives

$$
\partial_{t} M+u \cdot \nabla M=[\nabla u] M, \quad \partial_{t} M^{T}+u \cdot \nabla\left(M^{T}\right)=M^{T}[\nabla u]^{T} .
$$

Using the relations (5.9), we get

$$
\partial_{t} \mathcal{A}+u \cdot \nabla \mathcal{A}=[\nabla u] \mathcal{A}+\mathcal{A}[\nabla u]^{T},
$$

where the initial condition is given by $\mathcal{A}(0)=\mathbb{I}-n_{0} \otimes n_{0}$. Note that $B$ verifies the same equation (5.8) with a different initial condition $B(0)=\mathbb{I}$. Following (5.10), we get

$$
\partial_{t} \operatorname{Tr}(\mathcal{A})+u \cdot \nabla \operatorname{Tr}(\mathcal{A})=2[\nabla u]: \mathcal{A}
$$

and

$$
\partial_{t} \operatorname{Tr}(\operatorname{Cof}(\mathcal{A}))+u \cdot \nabla \operatorname{Tr}(\operatorname{Cof}(\mathcal{A}))=2\left[\mathcal{A} \operatorname{Tr}(\mathcal{A})-\mathcal{A}^{2}\right]:[\nabla u]
$$

Using (5.12), we get

$$
\partial_{t} Z_{1}+u \cdot \nabla Z_{1}=Z_{1} \frac{\left[\mathcal{A} \operatorname{Tr}(\mathcal{A})-\mathcal{A}^{2}\right]}{\operatorname{Tr}(\operatorname{Cof}(\mathcal{A}))}:[\nabla u]
$$

We show in Lemma B.1 of Appendix B that $\mathcal{A} \operatorname{Tr}(\mathcal{A})-\mathcal{A}^{2}=\operatorname{Tr}(\operatorname{Cof}(\mathcal{A}))[\mathbb{I}-n \otimes n]$. Using (5.11) and the equation on $Z_{1}$, we get the equation for $Z_{2}$. We have that $\mathcal{C}_{i} n=0$ because $\mathcal{A} n=0$ (see 4.4). We will prove in Proposition B.3 of Appendix B that $Z_{1}$ captures the local area variation. In Appendix A, we provide many illustrations to explain why $Z_{2}$ is a relevant measure of shear variation. 
5.3. Computation of the elastic forces $F_{i}$. We introduce the regularized energy

$$
\mathcal{E}_{i}=\int_{\Omega} E_{i}\left(Z_{i}\right) \frac{1}{\varepsilon} \zeta\left(\frac{\phi}{\varepsilon}\right) \mathrm{d} x
$$

Here, $E_{i}$ is the constitutive law associated to the invariant $Z_{i}$.

Proposition 5.2. The time variation of $\mathcal{E}_{i}$, using the principle of virtual power

$$
\partial_{t} \mathcal{E}_{i}=-\int_{\Omega} F_{i} \cdot u \mathrm{~d} x
$$

corresponds to the following force:

$$
F_{i}=\nabla\left(E_{i}\left(Z_{i}\right) \frac{1}{\varepsilon} \zeta\left(\frac{\phi}{\varepsilon}\right)\right)+\operatorname{div}\left(E_{i}^{\prime}\left(Z_{i}\right) Z_{i} \mathcal{C}_{i} \frac{1}{\varepsilon} \zeta\left(\frac{\phi}{\varepsilon}\right)\right)
$$

Proof. The derivative with respect to $t$ gives

$$
\partial_{t} \mathcal{E}_{i}=\int_{\Omega} E_{i}^{\prime}\left(Z_{i}\right)\left(Z_{i}\right)_{t} \frac{1}{\varepsilon} \zeta\left(\frac{\phi}{\varepsilon}\right) \mathrm{d} x+\int_{\Omega} E_{i}\left(Z_{i}\right) \frac{1}{\varepsilon^{2}} \zeta^{\prime}\left(\frac{\phi}{\varepsilon}\right) \phi_{t} \mathrm{~d} x
$$

Using the transport equation on $\phi$ and (5.5), (5.6) we get

$$
\begin{aligned}
\partial_{t} \mathcal{E}_{i}=\int_{\Omega} E_{i}^{\prime}\left(Z_{i}\right)\left(-u \cdot \nabla Z_{i}+[\nabla u]: Z_{i} \mathcal{C}_{i}\right) \frac{1}{\varepsilon} \zeta\left(\frac{\phi}{\varepsilon}\right) \mathrm{d} x & \\
& +\int_{\Omega} E_{i}\left(Z_{i}\right) \frac{1}{\varepsilon^{2}} \zeta^{\prime}\left(\frac{\phi}{\varepsilon}\right)(-u \cdot \nabla \phi) \mathrm{d} x .
\end{aligned}
$$

Integrating the second term by parts gives the following (the integral on $\partial \Omega$ vanishes since $\zeta\left(\frac{\phi}{\varepsilon}\right)=0$ on $\left.\partial \Omega\right)$ :

$$
\begin{aligned}
\partial_{t} \mathcal{E}_{i}=-\int_{\Omega} u \cdot \nabla\left(E_{i}\left(Z_{i}\right)\right) \frac{1}{\varepsilon} \zeta\left(\frac{\phi}{\varepsilon}\right)+\operatorname{div}\left(E_{i}^{\prime}\left(Z_{i}\right) Z_{i} \mathcal{C}_{i} \frac{1}{\varepsilon} \zeta\left(\frac{\phi}{\varepsilon}\right)\right) \cdot u & \\
& +E_{i}\left(Z_{i}\right) u \cdot \nabla\left(\frac{1}{\varepsilon} \zeta\left(\frac{\phi}{\varepsilon}\right)\right) \mathrm{d} x
\end{aligned}
$$

Grouping the first and last term and using (5.14) leads to the expression (5.15).

In the incompressible case, the gradient can be forgotten up to a redefinition of pressure and $Z_{1}=|\nabla \phi|$ thanks to (B.1). Therefore, we find the previous result (5.2). We have that

$$
\operatorname{div}\left(E_{i}^{\prime}\left(Z_{i}\right) Z_{i} \mathcal{C}_{i} \frac{1}{\varepsilon} \zeta\left(\frac{\phi}{\varepsilon}\right)\right)=\operatorname{div}\left(E_{i}^{\prime}\left(Z_{i}\right) Z_{i} \mathcal{C}_{i}\right) \frac{1}{\varepsilon} \zeta\left(\frac{\phi}{\varepsilon}\right)+E_{i}^{\prime}\left(Z_{i}\right) Z_{i} \mathcal{C}_{i} n \frac{|\nabla \phi|}{\varepsilon^{2}} \zeta^{\prime}\left(\frac{\phi}{\varepsilon}\right)
$$

The last term vanishes because $\mathcal{C}_{i} n=0$. With the identity $\operatorname{div}(A v)=\operatorname{div}\left(A^{T}\right) \cdot v+A^{T}$ : $\nabla v$ and the symmetry of $\mathcal{C}_{i}$, we get

$$
\operatorname{div}\left(E_{i}^{\prime}\left(Z_{i}\right) Z_{i} \mathcal{C}_{i} n\right)=0=\operatorname{div}\left(E_{i}^{\prime}\left(Z_{i}\right) Z_{i} \mathcal{C}_{i}\right) \cdot n+E_{i}^{\prime}\left(Z_{i}\right) Z_{i} \mathcal{C}_{i}:[\nabla n]
$$


With the definition of $\mathcal{C}_{1}$ in (5.5) and with (5.6), we find the normal part of forces $F_{i}$ (without the pressure term):

$$
F_{1} \cdot n=-\operatorname{Tr}([\nabla n]) E_{1}^{\prime}\left(Z_{1}\right) Z_{1} \frac{1}{\varepsilon} \zeta\left(\frac{\phi}{\varepsilon}\right)
$$

With the relation $2 Z_{1} Z_{2}=\operatorname{Tr}(\mathcal{A})($ see $(4.6))$, we get

$$
F_{2} \cdot n=-(2 \operatorname{Tr}(\mathcal{A}[\nabla n])-\operatorname{Tr}(\mathcal{A}) \operatorname{Tr}([\nabla n])) \frac{E_{2}^{\prime}\left(Z_{2}\right)}{2 Z_{1}} \frac{1}{\varepsilon} \zeta\left(\frac{\phi}{\varepsilon}\right) .
$$

In the previous formulas we used the relation $[\mathbb{I}-n \otimes n]:[\nabla n]=\operatorname{Tr}([\nabla n])$ (where $[\nabla n]^{T} n=0$ since $|n|^{2}=1$ ) which is by definition the surface mean curvature. Hence the normal force associated to the area variation (5.16) only depends on the geometry through the surface curvature. The normal force associated to the shear variation $(5.17)$ not only depends on the geometry but also on the deformations on the surface through $\mathcal{A}$. In the general case, the normal part of the shear force does not vanish.

5.4. Numerical method. The fluid-structure equations (5.3) and (5.4) are discretized with finite difference schemes on a staggered grid (see Figure 5.1 for a 2D configuration) with the classical projection method.

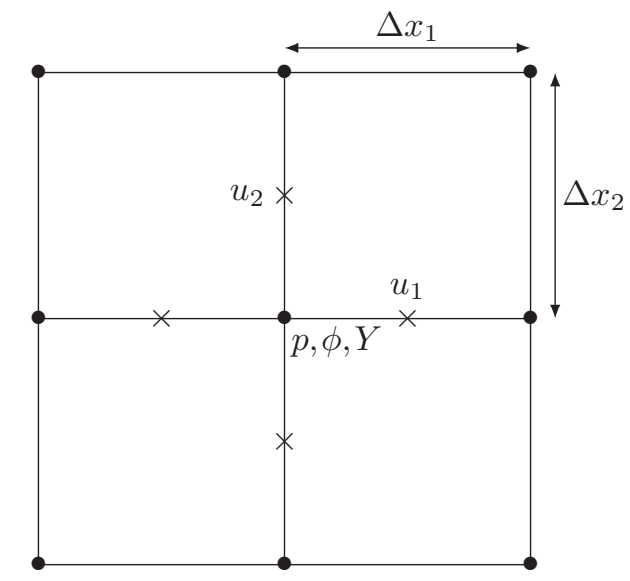

Fig. 5.1: Staggered grid with position of unknowns.

Let $\Delta t$ be the time step and $u^{n}, p^{n}, \phi^{n}, Y^{n}$ the time discretization of the variables at $t_{n}=n \Delta t$. The semi-discretization in time is given by

Step 1: $\quad \frac{u^{\star}-u^{n}}{\Delta t}+u^{n} \cdot \nabla u^{n}-\frac{1}{R_{e}} \Delta u^{n}=F_{1}\left(\phi^{n}, Y^{n}\right)+F_{2}\left(\phi^{n}, Y^{n}\right)$

Step 2: $\quad \Delta p^{n+1}=\frac{\operatorname{div}\left(u^{\star}\right)}{\Delta t}$

Step 3: $\quad u^{n+1}=u^{\star}-\Delta t \nabla p^{n+1}$

Step 4: $\frac{\phi^{n+1}-\phi^{n}}{\Delta t}+u^{n+1} \cdot \nabla \phi^{n}=0 \quad \frac{Y^{n+1}-Y^{n}}{\Delta t}+u^{n+1} \cdot \nabla Y^{n}=0$

In Step 1, a prediction of the velocity is computed with an explicit Euler scheme in time and classical central staggered velocity-pressure schemes of order two for the 
convection, the diffusion, and the source terms. An explicit scheme in time is used for the diffusion because the Reynolds number is large enough. In Step 2, the Poisson equation for the pressure is performed with the Fishpack library [1] (we use homogeneous Neumann boundary conditions). In Step 3, the the velocity is corrected to enforce the incompressibility condition. In Step 4, the transport equations are discretiezd with an explicit Euler scheme in time and a WENO5 scheme in space [14].

We do not perform the redistancing. Instead, we use the renormalization $\frac{\phi}{|\nabla \phi|}$ to measure the distance to interface. Thus, $|\nabla \phi| \frac{1}{\varepsilon} \zeta\left(\frac{\phi}{\varepsilon}\right)$ is replaced by $\frac{1}{\varepsilon} \zeta\left(\frac{\phi}{|\nabla \phi| \varepsilon}\right)$. This approach was proved in [9] to be efficient from the point of view of both volume conservation and interface force calculations. For the cut-off function, we considered the expression $\zeta(r)=\frac{1}{2}(1+\cos (\pi r))$ on $[-1,1]$ and $\zeta(r)=0$ elsewhere. We use in our simulations the linear elastic laws

$$
E_{1}^{\prime}(r)=\lambda(r-1) \quad E_{2}^{\prime}(r)=\mu(r-1),
$$

where $\lambda$ and $\mu$ are the elastic stretching and shear modulus (however the model is still strongly nonlinear due to the geometric nonlinearities and the coupling with NavierStokes equations).

5.5. Numerical tests. In this section, we provide evidences of numerical convergence of our method on the illustrative test case of a sheared elastic sphere. The domain $Q=[-1,1]^{3}$ is discretized on a Cartesian mesh with 128 points in each direction. We choose in our simulation a Reynolds number $R_{e}=100$, an elastic stretching modulus $\lambda=1$, and a shear modulus $\mu=0.1$. The parameter $\varepsilon$ is equal to $3.5 \Delta x$ in the simulations where $\Delta x$ is the grid size. We take the time step $\Delta t=1.310^{-3} s$. We impose zero velocity for the initial and boundary conditions. The immersed initial surface is a sphere, so that

$$
\phi_{0}(x, y, z)=\sqrt{x^{2}+y^{2}+z^{2}}-0.5
$$

but which is not in its rest state, since material points have been moved, so that

$$
Y(x, y, z, 0)=\left(x \cos \left(t_{0} z\right)+y \sin \left(t_{0} z\right),-x \sin \left(t_{0} z\right)+y \cos \left(t_{0} z\right), z\right) .
$$

This corresponds to a deformation of the sphere when a 3D circular shear (see the expression of $Y$ for TC4 in Table A.1 and Figure A.8) is applied on opposite directions on the north and south poles (with respect to the $z$-axis) until $t=t_{0}$. Here, we take $t_{0}=\pi$. While this initial deformation was (artificially) imposed with no area variation (actually, the sheared surface is still geometrically a sphere), the area will change when it starts to relax, so the force $F_{1}$ is also involved. The motion is however driven by $F_{2}$. The numerical results at different time steps are presented in Figure 5.2. For plotting purposes, we represented on the deformed surface a checker board pattern which was tracked with markers to visualize the shear relaxation. In Figure 5.3, we plot the velocity magnitude at $t=0.5 \mathrm{~s}$ while the relaxation takes place. Due to the large imposed shear, the surface undergoes a complex deformation involving some small folds. These kinds of ripples were also observed in [28] in the simulation of a capsule in a simple shear flow. A nice feature of our numerical code is its ability to relax toward a stable solution without any curvature energy. Some grid effects are, however, present for large time (see the last image of Figure 5.2). The use of an unstructured mesh and finite-element solver to remove this effect is presently under study. 

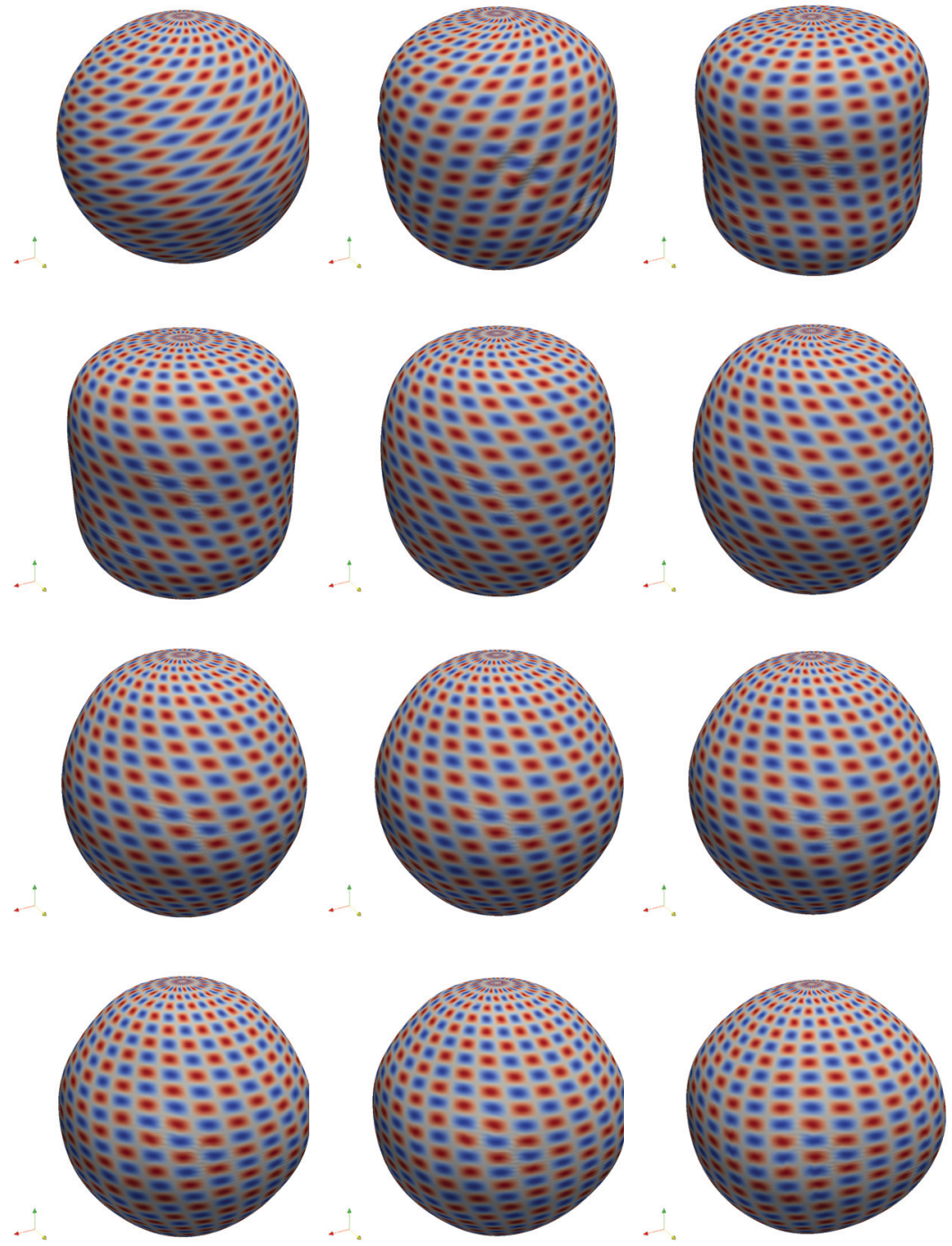

Fig. 5.2: Numerical simulation of the relaxation of a sphere due to shear force at times $t=0,0.5 s, 1 s, 1.5 s, 2 s, 2.5 s, 3 s, 3.5,4 s, 4.5 s, 5 s, 9 s$. Lagrangian markers are used for plotting a checkered pattern coming back to an undeformed state when shear decreases.

To provide more quantitative results, we plotted in Figure 5.4 and Figure 5.5 pressure slices following the $x$ - and $z$-axes during this relaxation for $t=0.1$ and $t=1.2$. The computations were performed with $N$ points in each direction for three resolutions with $N=64,128,256$. Accordingly, we chose $\varepsilon=1.75 \Delta x, 3.5 \Delta x, 7 \Delta x$, where $\Delta x$ is the corresponding grid size, so that the numerical interface width remains constant. We observe numerical convergence. Likewise, Figure 5.6 depicts the variation of vertical radius through time.

As the flow is incompressible, the map $Y(\cdot, t): \Omega \rightarrow \Omega$ conserves volume; that is, $\operatorname{det} \nabla Y=1$ in the continuous case. However, after time and space discretization, and due to numerical errors introduce while solving for the transport equations on $Y$, such a 


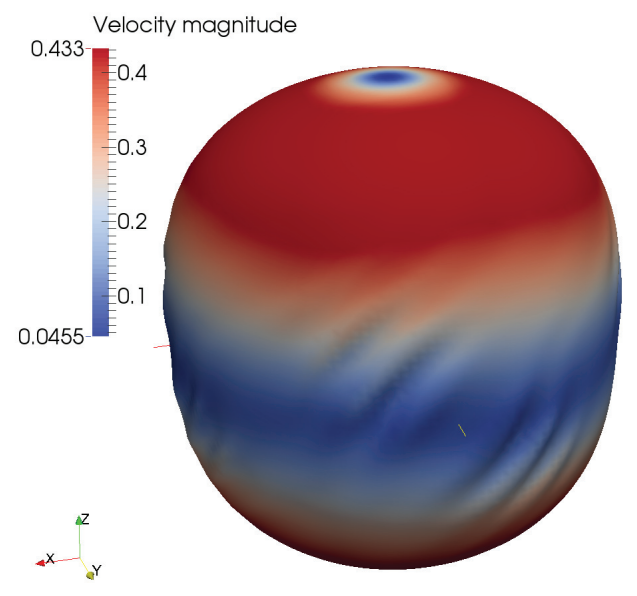

Fig. 5.3: Velocity magnitude at $t=0.5 s$.
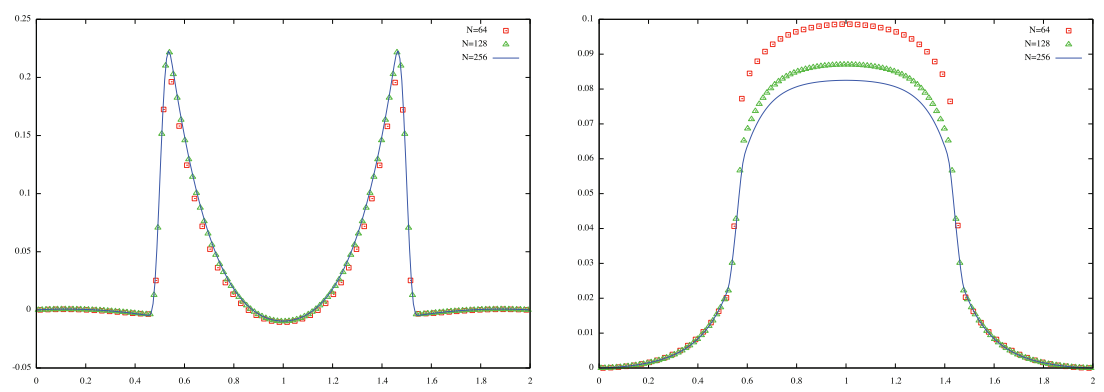

Fig. 5.4: Pressure along the $x$-axis at time $t=0.1$ (Left); and $t=1.2$ (Right) for $N=64,128,256$.
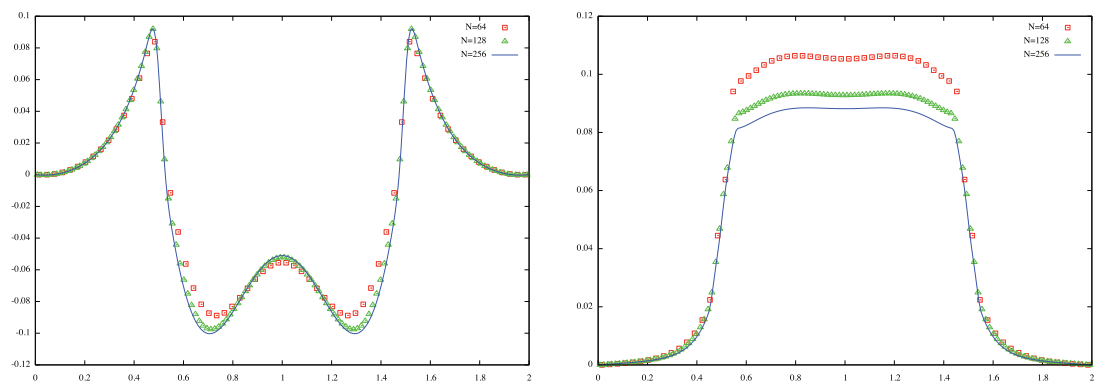

Fig. 5.5: Pressure along the $z$-axis at time $t=0.1$ (Left) and $t=1.2$ (Right) for $N=64,128,256$.

nonlinear combination of derivatives will not fully respect this constraint. On Figure 5.7 and Figure 5.8, we depict the RMS (Root Mean Square) of $\operatorname{det} \nabla Y-1$ as a function of time, both for the whole domain and on the interface. Thus, the plotted quantities are, respectively,

$$
R M S_{\Omega}(t)=\left(\frac{1}{|\Omega|} \int_{\Omega}|\operatorname{det} \nabla Y(x, t)-1|^{2} \mathrm{~d} x\right)^{\frac{1}{2}}
$$



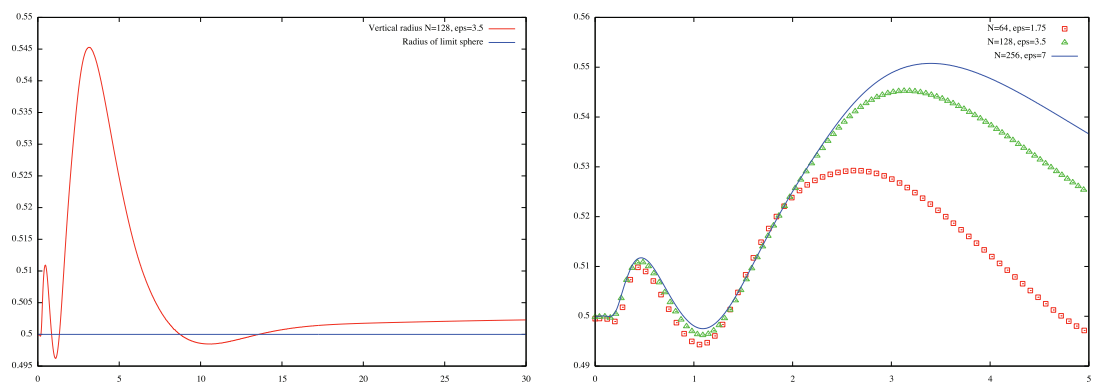

Fig. 5.6: Vertical radius up to $t=30$ (Left); zoomed to $t \in[0,5]$ for $N=64,128,256$.(Right).

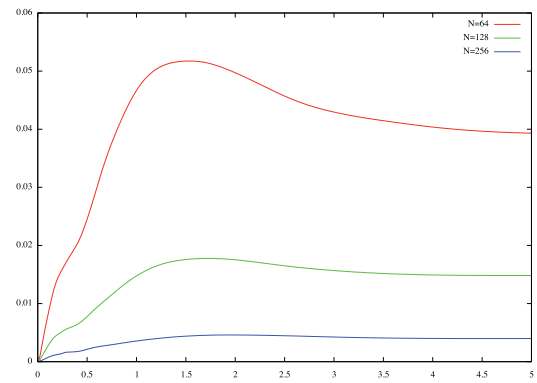

Fig. 5.7: Time evolution for $N=64,128,256$ of $t \rightarrow R M S_{\Omega}(t)$ defined in (5.21).

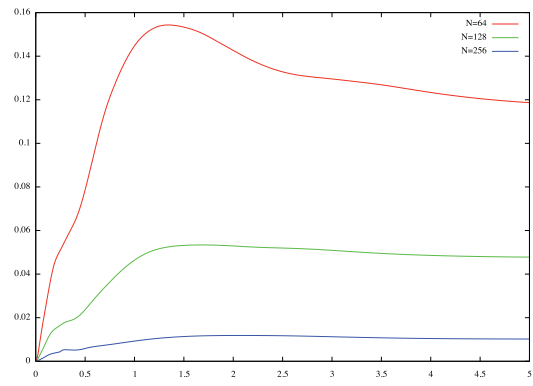

Fig. 5.8: footnotesize Time evolution, for $N=64,128,256$ of $t \rightarrow R M S_{\Gamma_{t}}(t)$ defined in (5.21).

and

$$
R M S_{\Gamma_{t}}(t)=\left(\frac{1}{\left|\Gamma_{t}\right|} \int_{\Gamma_{t}}|\operatorname{det} \nabla Y(x, t)-1|^{2} \mathrm{~d} s\right)^{\frac{1}{2}} .
$$

While $N=64$ is clearly under-resolved, the figures show numerical convergence of those RMS as $N$ increases.

\section{Conclusion}

In this paper, we introduced a way to account for membrane shear in a completely Eulerian framework. The idea was to build two invariants from algebraic combinations of the space derivatives of the backward characteristics of the continuous medium velocity field. The first invariant accounts for changes of local area of the membrane, while the second records the local membrane shear. We showed how to compute those invariants in an Eulerian way and produced some evidences that the second invariant indeed captures 
the membrane shear. The fluid-structure coupling is therefore rephrased as a NavierStokes equation with source term involving the two invariants, coupled to a vectorial transport equation for the backward characteristics. The formulation is therefore very versatile since it could be implemented as an add-on to a preexisting fluid solver. In this first work, we presented outputs of a 3D numerical code using finite differences and a projection method on a staggered mesh, which simulate the relaxation of a spherical membrane which has been sheared from its poles. Our model and code are able to capture ripples due to the bucking of membrane in a stable way without any extra smoothing curvature energy. Future work will focus on the application of this method to model red blood cells and capsules in 3D flows.

\section{Appendix A. Analytical illustrations for $Z_{2}$.}

We present some analytical illustrations to show that $Z_{2}$ is intuitively a good measure of the local shear variation of a surface. In all the test cases, we define an initial surface $\Gamma_{0}=\left\{\phi_{0}=0\right\}$ and a velocity field $u$ which will move material points of this surface. Then we compute the backward characteristics $Y$ and the invariants $Z_{1}$ and $Z_{2}$ to see how these Eulerian quantities could record this motion. The test cases and the results are described in Table A.1.

\begin{tabular}{|c|c|c|c|c|c|}
\hline TC & $\phi_{0}(x, y, z)$ & $u$ & $Y(x, y, z, t)$ & $Z_{1}$ & $Z_{2}$ \\
\hline 1 & $\mathrm{z}$ & $\left(\begin{array}{c}x \\
\alpha y \\
0\end{array}\right)$ & $\left(\begin{array}{c}x e^{-t} \\
y e^{-\alpha t} \\
z\end{array}\right)$ & $e^{t(1+\alpha)}$ & $\operatorname{ch}(t(1-\alpha))$ \\
\hline 2 & $\mathrm{z}$ & $\left(\begin{array}{c}\beta y \\
x \\
0\end{array}\right)$ & $\left(\begin{array}{c}x \operatorname{ch}(t \sqrt{\beta})-y \sqrt{\beta} \operatorname{sh}(t \sqrt{\beta}) \\
-\frac{\sqrt{\beta}}{\sqrt{\beta}} \operatorname{sh}(t \sqrt{\beta})+y \operatorname{ch}(t \sqrt{\beta}) \\
z\end{array}\right)$ & 1 & $1+\frac{(1+\beta)^{2}}{2 \beta} \operatorname{sh}^{2}(t \sqrt{\beta})$ \\
\hline 3 & $x^{2}+y^{2}-1$ & $\left(\begin{array}{c}-y z \\
x z \\
0\end{array}\right)$ & $\left(\begin{array}{c}x \cos (t z)+y \sin (t z) \\
-x \sin (t z)+y \cos (t z) \\
z\end{array}\right)$ & 1 & $1+\frac{t^{2}}{2}\left(x^{2}+y^{2}\right)$ \\
\hline 4 & $x^{2}+y^{2}+z^{2}-1$ & $\left(\begin{array}{c}-y z \\
x z \\
0\end{array}\right)$ & $\left(\begin{array}{c}x \cos (t z)+y \sin (t z) \\
-x \sin (t z)+y \cos (t z) \\
z\end{array}\right)$ & 1 & $1+\frac{t^{2}\left(x^{2}+y^{2}\right)^{2}}{2\left(x^{2}+y^{2}+z^{2}\right)}$ \\
\hline \hline 5 & $x^{2}+y^{2}+z^{2}-1$ & $\left(\begin{array}{c}x \\
y \\
z\end{array}\right)$ & $\left(\begin{array}{c}e^{-t} x \\
e^{-t} y \\
e^{-t} z\end{array}\right)$ & $e^{2 t}$ & 1 \\
\hline 6 & $\max (|x|,|y|,|z|)-1$ & $\left(\begin{array}{c}0 \\
x \\
0\end{array}\right)$ & $\left(\begin{array}{c}x \\
y-t x \\
z\end{array}\right)$ & \multicolumn{2}{|c|}{ See (A.1)-(A.3) } \\
\hline 7 & $x^{2}+y^{2}+z^{2}-1$ & $\left(\begin{array}{c}0 \\
x \\
0\end{array}\right)$ & $\left(\begin{array}{c}x \\
y-t x \\
z\end{array}\right)$ & See & \multicolumn{2}{|c|}{ See (A.5) } \\
\hline
\end{tabular}

Table A.1: Table of test cases.

A.1. Deformations where $\Gamma_{t}=\Gamma_{0}$. In this first series of test cases (TC1 to TC4), while material points could have moved, globally the initial and deformed surfaces are the same $\left(\Gamma_{0}=\Gamma_{t}\right)$. Moreover, the deformations are uniform in space in the sense that the invariants $Z_{i}$ on the surface do not depend on the spatial variables (except for TC4).

What we refer to as the $\alpha$ and $\beta$ deformations (TC1 and TC2) are 2D, and the initial surface is the plane $z=0$. The velocity field of each deformation is plotted in the figures below with the corresponding values of $Z_{1}$ and $Z_{2}$. The $\beta=-1$ deformation is a rotation, and, as expected (slipping from hyperbolic to circular functions with $\sqrt{\beta}$ identified to $i \in \mathbb{C}$ ), there is no area variation and shear variation (see Figure A.1). 


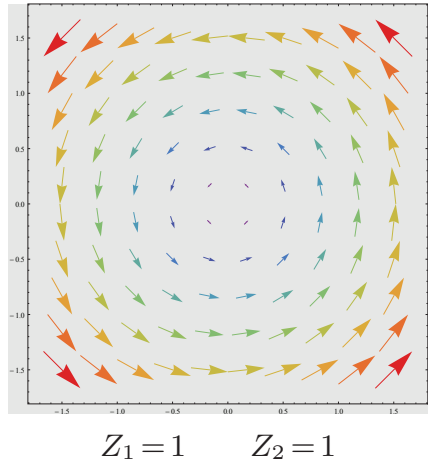

Fig. A.1: Velocity field $(-y, x, 0)$ of the $\beta=-1$ deformation (TC2).

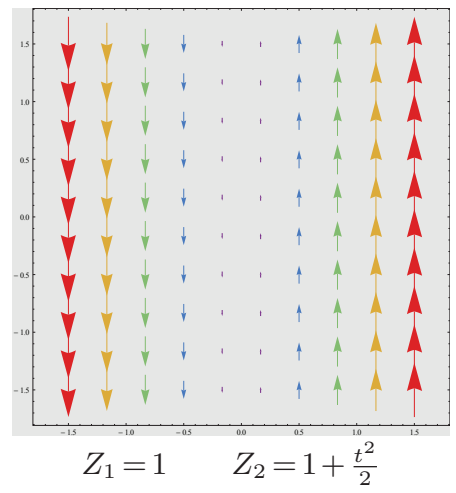

Fig. A.3: Velocity field $(0, x, 0)$ of the $\beta=0$ deformation (TC2).

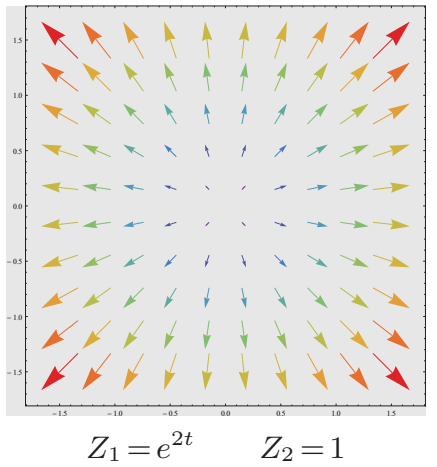

Fig. A.2: Velocity field $(x, y, 0)$ of the $\alpha=1$ deformation (TC1).

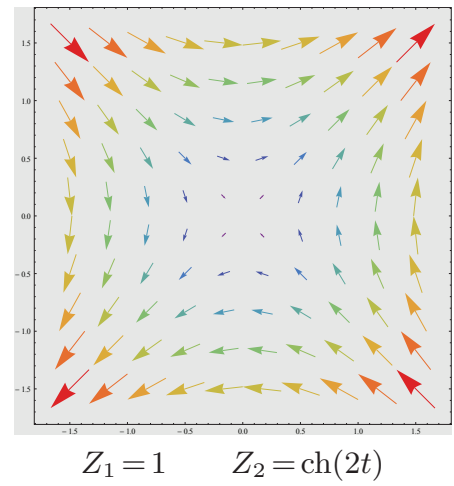

Fig. A.4: Velocity field $(y, x, 0)$ of the $\beta=1$ deformation (TC2).

The $\alpha=1$ deformation is a pure dilatation, and, as expected, there is only area variation (see Figure A.2). The $\beta=0, \beta=1$, and $\alpha=-1$ deformations correspond to different shear transformations, and, as expected, there is only shear variation (see figures A.3, A.4, and A.5). Note that, for the $\beta=0$ test case, we take the limit $\beta \longrightarrow 0$ the results for $Y$ and $Z_{i}$. Moreover, for the $\beta=0$ deformation, we found the same invariants as in the Lagrangian case (see (3.3)). The $\alpha=0$ deformation is an uniaxial deformation and there is area and shear variation (see Figure A.6). It can be quite surprising at first sight, but, when a surface is stretched in two directions with different magnitude $(\alpha \neq 1)$, we do have shear and therefore as expected $Z_{2}=\operatorname{ch}(t(1-\alpha)) \neq 1$. In the "3D circular shear" test cases the initial surfaces are a cylinder (TC3 see Figure A.7) and a sphere (TC4 see Figure A.8). In each plane $\{z=\alpha\}$, the velocity is a rotation of magnitude $\alpha$. In these test cases, there is no area variation but pure shear variation. For TC3, $Z_{2}=1+\frac{t^{2}}{2}$ is constant on the surface $\left(x^{2}+y^{2}=1\right.$ on the cylinder). This test case is clearly a $3 \mathrm{D}$ generalization on a cylinder of the $2 \mathrm{D} \beta=0$ deformation, and this is why we found the same invariants. For TC4, $Z_{2}=1+\frac{t^{2}}{2}\left(1-z^{2}\right)^{2}$ on the surface and depends only on the height $z$ which is intuitive $\left(x^{2}+y^{2}+z^{2}=1\right.$ on the sphere). 


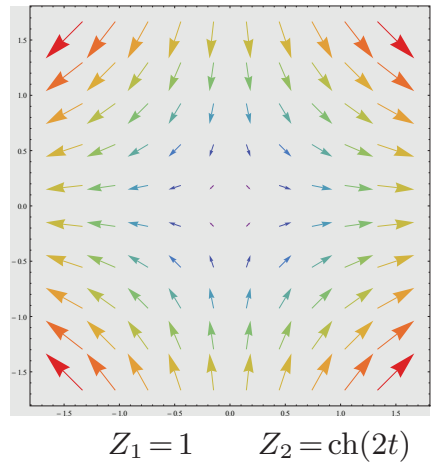

Fig. A.5: Velocity field $(x,-y, 0)$ of the $\alpha=-1$ deformation (TC1).

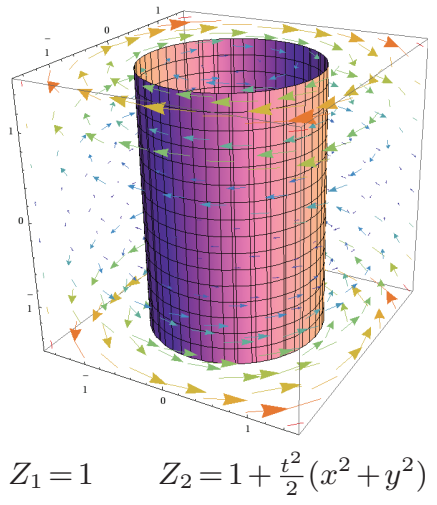

Fig. A.7: Velocity field $(-y z, x z, 0)$ of the $3 D$ circular shear and initial shape of the cylinder (TC3).

A.2. Deformations where $\Gamma_{t} \neq \Gamma_{0}$. We present now three test cases (TC57) where the deformed surface $\Gamma_{t}$ is different from the initial shape $\Gamma_{0}$. For the $3 \mathrm{D}$ dilatation deformation (TC5 see Figure A.9), the initial surface is a sphere. In this test case we have only area variation, as expected.

In the last test cases, the same shear velocity field (see Figure A.10) is applied on a cube and on a sphere. For the test case TC6, the deformed shape of the cube (see Figure A.11) is given by the zero level set of

$$
\phi(x, y, z, t)=\max (|x|,|y-t x|,|z|)-1 .
$$

The calculations are done independently on each plane that composes the cube. We have the following results:

On the faces $\{x= \pm 1\}$

$Z_{1}=1$

$Z_{2}=1$

On the faces $\{y-t x= \pm 1\}$

$Z_{1}=\sqrt{1+t^{2}}$

$Z_{2}=\frac{2+t^{2}}{2 \sqrt{1+t^{2}}}$ 


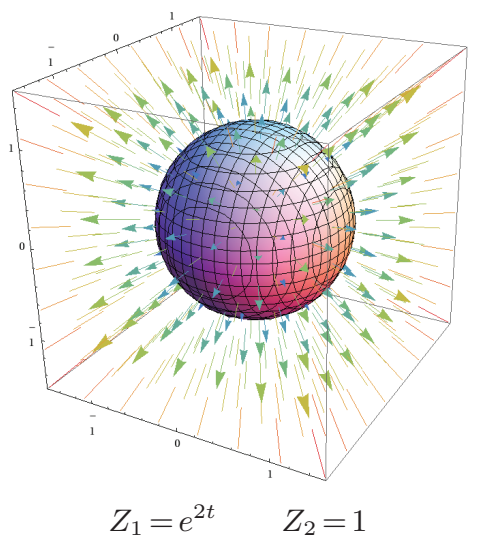

Fig. A.9: Velocity field $(x, y, z)$ of the 3D dilatation and initial shape of the sphere (TC5).

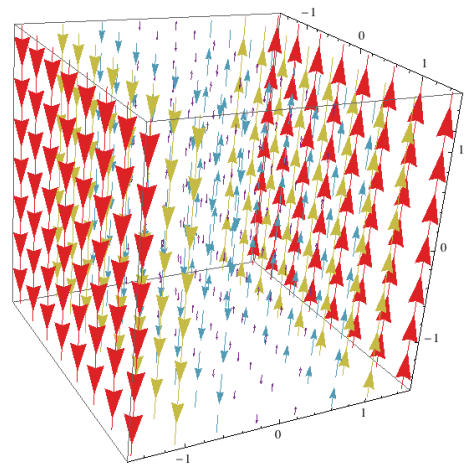

Fig. A.10: Velocity field $(0, x, 0)$ of the shear deformation(TC6-7).

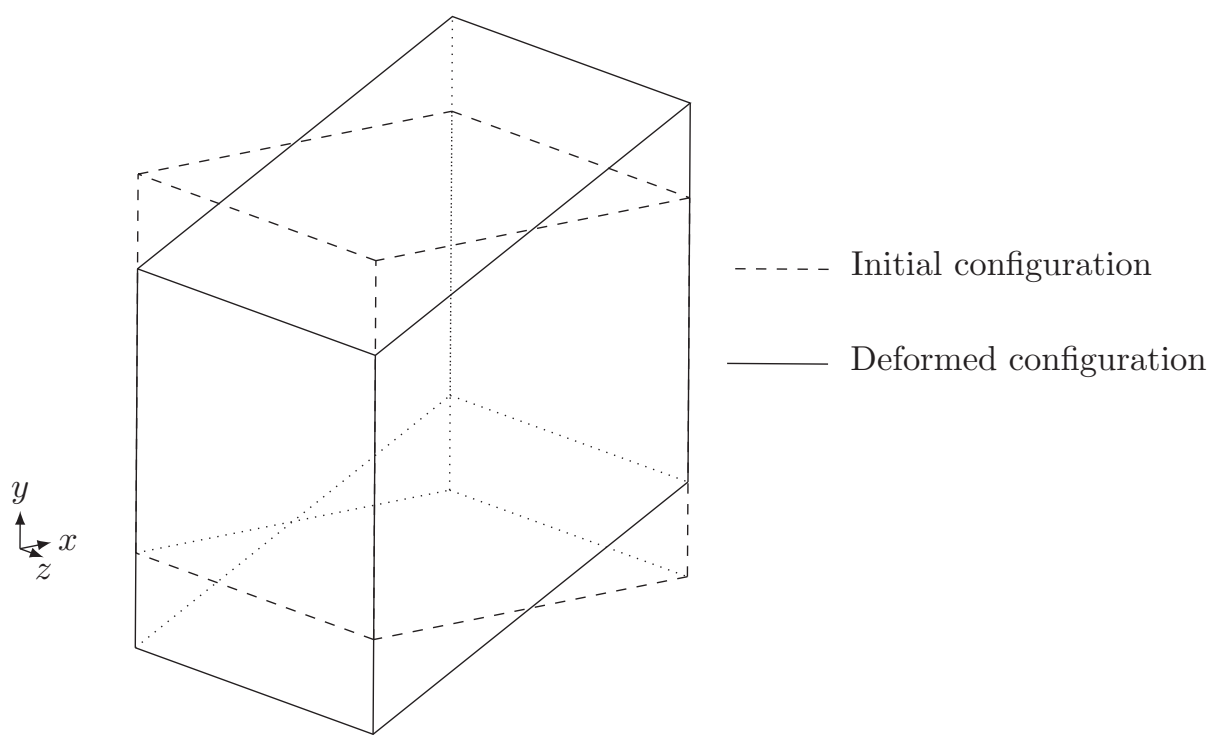

Fig. A.11: Initial and deformed shape of the cube (TC6). 

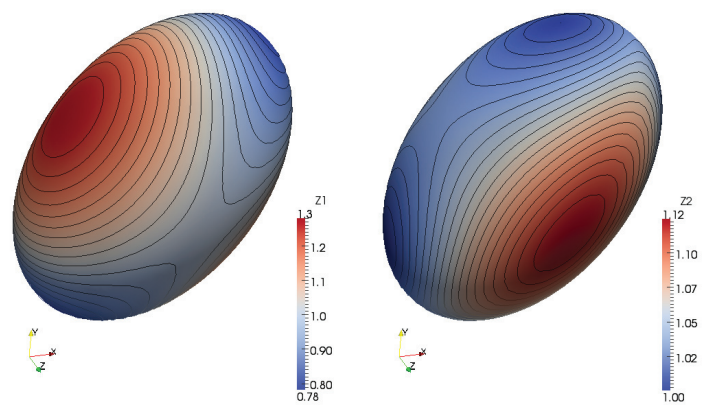

Fig. A.12: Plot of iso-contours of $Z_{1}$ and $Z_{2}$ on the deformed surface at $t=0.5 \mathrm{~s}$ (TC7).

$$
\text { On the faces }\{z= \pm 1\} \quad Z_{1}=1, \quad Z_{2}=1+\frac{t^{2}}{2}
$$

On the faces corresponding to $\{x= \pm 1\}$, there is no area and shear variation (the faces are just translated). For the faces of equations $\{y-t x= \pm 1\}$, the faces are stretched in only one direction and there is area and shear variation as in the $2 \mathrm{D} \alpha=0$ deformation. For the faces $\{z= \pm 1\}$, there is only shear variation as in the $2 \mathrm{D} \beta=0$ deformation.

For the test case TC7, the deformed shape of the sphere $\Gamma_{t}$ is given by the zero level set of

$$
\phi(x, y, z, t)=x^{2}+(y-t x)^{2}+z^{2}-1 .
$$

We have that

$$
\begin{gathered}
Z_{1}=\sqrt{1+t^{2}+\frac{t^{2}\left(x^{2}-z^{2}\right)-2 t x y}{x^{2}+(y-t x)^{2}+z^{2}}} \\
Z_{2}=\left(1+\frac{t^{2}}{2}+\frac{t^{2} x^{2}-2 t x y}{2\left(x^{2}+(y-t x)^{2}+z^{2}\right)}\right) \frac{1}{Z_{1}} .
\end{gathered}
$$

In Figure A.12, iso-contours of $Z_{1}$ and $Z_{2}$ are plotted on the deformed surface $\Gamma_{t}$. The results are intuitive: the area variation reaches its maximum on points that are orthogonal to the $y=t x$ plane while the shear variation is larger along the $z$-axis. The deformation and the initial surface are the same as in the Lagrangian test case in Section 3.3. In the formula (A.5), there are no discontinuities at the poles $\left(x^{2}+(y-t x)^{2}+z^{2}=1\right.$ on the surface $\Gamma_{t}$ ), unlike in the Lagrangian formula (3.6).

\section{Appendix B.}

\section{B.1. Technical lemma.}

LEMma B.1. The following identity holds:

$$
\mathcal{A} \operatorname{Tr}(\mathcal{A})-\mathcal{A}^{2}=\operatorname{Tr}(\operatorname{Cof}(\mathcal{A}))[\mathbb{I}-n \otimes n] .
$$

Proof. It is equivalent to prove the lemma in any basis of $\mathbb{R}^{3}$. We consider the orthonormal basis of $\mathbb{R}^{3} \mathcal{B}^{\prime}=\left(e_{1}^{\prime}, e_{2}^{\prime}, e_{3}^{\prime}\right)=\left(\tau_{1}, \tau_{2}, n\right)$, where $\left(\tau_{1}, \tau_{2}\right)$ is an orthonormal basis of the tangent plane orthogonal to $n$. Let $\mathcal{A}^{\prime}$ be the matrix of the tensor $\mathcal{A}$ in the basis $\mathcal{B}^{\prime}$, and we denote by $\mathcal{A}_{i j}^{\prime}$ its coefficients. We have with (4.4) that $\mathcal{A} n=0$; hence 
we have $\mathcal{A}_{i 3}^{\prime}=0$. Since $\mathcal{A}$ is a symmetric tensor and $\mathcal{B}^{\prime}$ is an orthonormal basis, the matrix $\mathcal{A}^{\prime}$ is symmetric, and its structure is given by

$$
\mathcal{A}^{\prime}=\left(\begin{array}{ccc}
\mathcal{A}_{11}^{\prime} & \mathcal{A}_{12}^{\prime} & 0 \\
\mathcal{A}_{12}^{\prime} & \mathcal{A}_{22}^{\prime} & 0 \\
0 & 0 & 0
\end{array}\right)
$$

and therefore

$$
\left(\mathcal{A}^{\prime}\right)^{2}=\left(\begin{array}{ccc}
\left(\mathcal{A}_{11}^{\prime}\right)^{2}+\left(\mathcal{A}_{12}^{\prime}\right)^{2} & \mathcal{A}_{11}^{\prime} \mathcal{A}_{12}^{\prime}+\mathcal{A}_{12}^{\prime} \mathcal{A}_{22}^{\prime} & 0 \\
\mathcal{A}_{11}^{\prime} \mathcal{A}_{12}^{\prime}+\mathcal{A}_{12}^{\prime} \mathcal{A}_{22}^{\prime} & \left(\mathcal{A}_{12}^{\prime}\right)^{2}+\left(\mathcal{A}_{22}^{\prime}\right)^{2} & 0 \\
0 & 0 & 0
\end{array}\right)
$$

In the basis $\mathcal{B}^{\prime}$, we have

$$
\mathbb{I}-n \otimes n=\left(\begin{array}{lll}
1 & 0 & 0 \\
0 & 1 & 0 \\
0 & 0 & 0
\end{array}\right)
$$

$\mathcal{A}^{\prime}$ and $\mathcal{A}$ have the same invariants so

$$
\begin{aligned}
\operatorname{Tr}(\mathcal{A}) & =\operatorname{Tr}\left(\mathcal{A}^{\prime}\right)=\mathcal{A}_{11}^{\prime}+\mathcal{A}_{22}^{\prime} \\
\operatorname{Tr}(\operatorname{Cof}(\mathcal{A})) & =\operatorname{Tr}\left(\operatorname{Cof}\left(\mathcal{A}^{\prime}\right)\right)=\mathcal{A}_{11}^{\prime} \mathcal{A}_{22}^{\prime}-\left(\mathcal{A}_{12}^{\prime}\right)^{2} .
\end{aligned}
$$

It is now easy to check that $\mathcal{A}^{\prime} \operatorname{Tr}\left(\mathcal{A}^{\prime}\right)-\left(\mathcal{A}^{\prime}\right)^{2}=\operatorname{Tr}\left(\operatorname{Cof}\left(\mathcal{A}^{\prime}\right)\right)[\mathbb{I}-n \otimes n]$, and the lemma is proved.

B.2. Equivalent expression for $Z_{1}$. In this subsection, we give an equivalent expression of $Z_{1}$ which links the present formulation with our previous work [9].

Proposition B.2. Let $J=\operatorname{det}\left(\nabla_{\xi} X\right)=\operatorname{det}(\nabla Y)^{-1}$ be the volume ratio. Then

$$
Z_{1}=J \frac{|\nabla \phi|}{\left|\nabla \phi_{0}(Y)\right|}
$$

Proof. The invariant $Z_{1}$ is defined by (4.5)

$$
Z_{1}=\sqrt{\operatorname{Tr}(\operatorname{Cof}(\mathcal{A}))}
$$

With the definition (4.3), we get

and

$$
\operatorname{Tr}(\mathcal{A})^{2}=\left(\operatorname{Tr}(B)-\frac{\left(B^{2} n\right) \cdot n}{(B n) \cdot n}\right)^{2}
$$

$$
\operatorname{Tr}\left(\mathcal{A}^{2}\right)=\operatorname{Tr}\left(B^{2}\right)-2 \frac{\left(B^{3} n\right) \cdot n}{(B n) \cdot n}+\left(\frac{\left(B^{2} n\right) \cdot n}{(B n) \cdot n}\right)^{2} .
$$

Using the Cayley-Hamilton theorem $B^{3}-\operatorname{Tr}(B) B^{2}+\operatorname{Tr}(\operatorname{Cof}(B)) B-\operatorname{det}(B) \mathbb{I}=0$, we get

$$
\operatorname{Tr}(\operatorname{Cof}(\mathcal{A}))=\frac{1}{(B n) \cdot n}\left(\left(B^{3} n\right) \cdot n-\operatorname{Tr}(B)\left(B^{2} n\right) \cdot n+\operatorname{Tr}(\operatorname{Cof}(B))(B n) \cdot n\right)=\frac{\operatorname{det}(B)}{(B n) \cdot n} .
$$


The gradient of (2.5) gives the relation $\nabla \phi=[\nabla Y]^{T} \nabla \phi_{0}(Y)$, so

$$
(B n) \cdot n=\left|[\nabla Y]^{-T} \frac{\nabla \phi}{|\nabla \phi|}\right|^{2}=\frac{\left|\nabla \phi_{0}(Y)\right|^{2}}{|\nabla \phi|^{2}} .
$$

We have the relation $\operatorname{det}(B)=J^{2}$, so the expression of $Z_{1}$ (B.2) reduces to (B.1). This recovers the result of [9], where the area variation captured by $|\nabla \phi|$ in the incompressible case $(J=1)$.

\section{B.3. Proof that $Z_{1}$ is the local area variation.}

Proposition B.3. Let $\Gamma_{0}$ a smooth surface, deformed with smooth forward characteristics in $\Gamma_{t}=X\left(\Gamma_{0}, t\right)$. We denote by $u$ the associated smooth velocity field. Let a parametrization of the surface $\Gamma_{t}$ be given by $\left(\theta_{1}, \theta_{2}\right) \mapsto \Psi\left(\theta_{1}, \theta_{2}, t\right)$, where $\Psi: U \times \mathbb{R}^{+} \longrightarrow$ $\mathbb{R}^{3}$ is smooth on an open set $U$ of $\mathbb{R}^{2}$. The local area variation verifies

$$
\frac{\left|\Psi_{, 1}\left(\theta_{1}, \theta_{2}, t\right) \wedge \Psi_{, 2}\left(\theta_{1}, \theta_{2}, t\right)\right|}{\left|\Psi_{, 1}\left(\theta_{1}, \theta_{2}, 0\right) \wedge \Psi_{, 2}\left(\theta_{1}, \theta_{2}, 0\right)\right|}=\frac{Z_{1}\left(\Psi\left(\theta_{1}, \theta_{2}, t\right), t\right)}{Z_{1}\left(\Psi\left(\theta_{1}, \theta_{2}, 0\right), 0\right)}=\frac{Z_{1}(x, t)}{Z_{1}(Y(x, t), 0)}
$$

for $x=\Psi\left(\theta_{1}, \theta_{2}, t\right)$ and with the notation $\Psi_{, i}\left(\theta_{1}, \theta_{2}, t\right):=\partial_{\theta_{i}} \Psi\left(\theta_{1}, \theta_{2}, t\right)$.

Proof. Let $f: \mathbb{R}^{3} \times \mathbb{R}^{+} \longrightarrow \mathbb{R}$ be a smooth function. The Reynolds formula for surfaces reads

$$
\frac{\mathrm{d}}{\mathrm{dt}}\left(\int_{\Gamma_{t}} f \mathrm{~d} s\right)=\int_{\Gamma_{t}} \partial_{t} f+u \cdot \nabla f+f[\nabla u]:[\mathbb{I}-n \otimes n] \mathrm{d} s .
$$

Let $g: \mathbb{R}^{3} \longrightarrow \mathbb{R}$ be smooth function and let $f(x, t)=\frac{g(Y(x, t))}{Z_{1}(x, t)}$. The expression in the previous integral becomes

$$
\frac{1}{Z_{1}}\left(\partial_{t}(g(Y))+u \cdot \nabla(g(Y))\right)-\frac{g(Y)}{\left(Z_{1}\right)^{2}}\left(\partial_{t} Z_{1}+u \cdot \nabla Z_{1}-Z_{1}[\nabla u]:[\mathbb{I}-n \otimes n]\right) .
$$

The first term vanishes with (2.2). The second term is also zero with (5.5). Therefore,

$$
\frac{\mathrm{d}}{\mathrm{dt}}\left(\int_{\Gamma_{t}} \frac{g(Y(x, t))}{Z_{1}(x, t)} \mathrm{d} s\right)=0 .
$$

Since, $\Psi\left(\theta_{1}, \theta_{2}, 0\right)=Y\left(\Psi\left(\theta_{1}, \theta_{2}, t\right), t\right)$ the equation (B.5) becomes

$$
\begin{aligned}
& \int_{U} \frac{g\left(\Psi\left(\theta_{1}, \theta_{2}, 0\right)\right)}{Z_{1}\left(\Psi\left(\theta_{1}, \theta_{2}, t\right), t\right)}\left|\Psi_{, 1}\left(\theta_{1}, \theta_{2}, t\right) \wedge \Psi_{, 2}\left(\theta_{1}, \theta_{2}, t\right)\right| \mathrm{d} \theta_{1} \mathrm{~d} \theta_{2} \\
= & \int_{U} \frac{g\left(\Psi\left(\theta_{1}, \theta_{2}, 0\right)\right)}{Z_{1}\left(\Psi\left(\theta_{1}, \theta_{2}, 0\right), 0\right)}\left|\Psi_{, 1}\left(\theta_{1}, \theta_{2}, 0\right) \wedge \Psi_{, 2}\left(\theta_{1}, \theta_{2}, 0\right)\right| \mathrm{d} \theta_{1} \mathrm{~d} \theta_{2} .
\end{aligned}
$$

This result holds for all function $g$. Therefore, (B.3) holds, and $Z_{1}$ measures the local area variation (in the compressible and incompressible cases).

\section{REFERENCES}

[1] J.C. Adams, P.N. Swarztrauber, and R. Sweet, Fishpack - efficient FORTRAN subprograms for the solution of separable elliptic partial differential equations, http://www2.cisl.ucar.edu/resources/legacy/fishpack. 


\section{IMMERSED ELASTIC SURFACES WITH FULL MEMBRANE ELASTICITY}

[2] D. Barthes-Biesel, Motion of a spherical microcapsule freely suspended in a linear shear flow, Journal of Fluid Mechanics, 100(04), 831-853, 1980.

[3] D. Barthes-Biesel and J.M. Rallison, The time-dependent deformation of a capsule freely suspended in a linear shear flow, Journal of Fluid Mechanics, 113, 251-267, 1981.

[4] J. Thomas Beale and John Strain, Locally corrected semi-lagrangian methods for stokes flow with moving elastic interfaces, J. Comput. Phys., 227(8), 3896-3920, 2008.

[5] J Beaucourt, F Rioual, T. Séon, T. Biben, and C. Misbah, Steady to unsteady dynamics of a vesicle in a flow, Physical Review E, 69(1), 011906, 2004.

[6] Thierry Biben, Alexander Farutin, and Chaouqi Misbah, Three-dimensional vesicles under shear flow: Numerical study of dynamics and phase diagram, Physical Review E, 83(3), 031921, 2011.

[7] Thierry Biben, Klaus Kassner, and Chaouqi Misbah, Phase-field approach to three-dimensional vesicle dynamics, Physical Review E, 72(4), 041921, 2005.

[8] Georges-Henri Cottet and Emmanuel Maitre, A level-set formulation of immersed boundary methods for fluid-structure interaction problems, Comptes Rendus Mathematique, 338(7), 581586, 2004.

[9] Georges-Henri Cottet and Emmanuel Maitre, A level set method for fluid-structure interactions with immersed surfaces, Math. Models Meth. Appl. Sci., 16(03), 415-438, 2006.

[10] Georges-Henri Cottet, Emmanuel Maitre, and Thomas Milcent, Eulerian formulation and level set models for incompressible fluid-structure interaction, ESAIM: Mathematical Modelling and Numerical Analysis, 42(03), 471-492, 2008.

[11] Vincent Doyeux, Yann Guyot, Vincent Chabannes, Christophe Prudomme, and Mourad Ismail, Simulation of two-fluid flows using a finite element/level set method. Application to bubbles and vesicle dynamics, J. Comput. Appl. Math., 246, 251-259, 2013.

[12] Knut Erik Teigen, Peng Song, John Lowengrub, and Axel Voigt, A diffuse-interface method for two-phase flows with soluble surfactants, J. Comput. Phys., 230(2), 375-393, 2011.

[13] Yannick Gorsse, Angelo Iollo, Thomas Milcent, and Haysam Telib, A simple cartesian scheme for compressible multimaterials, J. Comput. Phys., 272, 772-798, 2014.

[14] G.S. Jiang and C.W. Shu, Efficient implementation of weighted ENO schemes, J. Comput. Phys., 1996.

[15] Ken Kamrin, Chris H Rycroft, and Jean-Christophe Nave, Reference map technique for finitestrain elasticity and fluid-solid interaction, Journal of the Mechanics and Physics of Solids, 60(11), 1952-1969, 2012.

[16] Haruhiko Kohno and Jean-Christophe Nave, A new method for the level set equation using a hierarchical-gradient truncation and remapping technique, Comput. Phys. Commun., 184(6), 1547-1554, 2013.

[17] Long Lee and Randall J LeVeque, An immersed interface method for incompressible navier-stokes equations, SIAM J. Sci. Comput., 25(3), 832-856, 2003.

[18] John S Lowengrub, Andreas Rätz, and Axel Voigt, Phase-field modeling of the dynamics of multicomponent vesicles: Spinodal decomposition, coarsening, budding, and fission, Phys. Review E, 79(3), 031926, 2009.

[19] Emmanuel Maitre, Thomas Milcent, Georges-Henri Cottet, Annie Raoult, and Yves Usson, Applications of level set methods in computational biophysics, Math. Comput. Modelling, 49(11), 2161-2169, 2009.

[20] Olivier Mercier and Jean-Christophe Nave, The characteristic mapping method for the linear advection of arbitrary sets, arXiv preprint arXiv:1309.2731, 2013.

[21] Charles S. Peskin, The immersed boundary method, Acta numerica, 11, 479-517, 2002.

[22] J.-P. Pons, Gerardo Hermosillo, Renaud Keriven, and O. Faugeras, Maintaining the point correspondence in the level set framework, J. Comput. Phys., 220(1), 339-354, 2006.

[23] Th Richter and Th Wick, Finite elements for fluid-structure interaction in ALE and fully Eulerian coordinates, Comput. Meth. Appl. Mech. Engineering, 199(41), 2633-2642, 2010.

[24] Thomas Richter, A fully Eulerian formulation for fluid-structure-interaction problems, J. Comput. Phys., 233, 227-240, 2013.

[25] R. Skalak, A. Tozeren, R.P. Zarda, and S. Chien, Strain energy function of red blood cell membranes, Biophysical Journal, 13(3), 245-264, 1973.

[26] Boris Valkov, Chris H. Rycroft, and Ken Kamrin, Eulerian method for fluid-structure interaction and submerged solid-solid contact problems, J. Appl. Mech., 82(4), 041011, 2015.

[27] J. Walter, A.V. Salsac, and D. Barthes-Biesel, Ellipsoidal capsules in simple shear flow: prolate versus oblate initial shapes, Journal of Fluid Mechanics, 676, 318-347, 2011.

[28] J. Walter, A.V. Salsac, D. Barthès-Biesel, and P. Le Tallec, Coupling of finite element and boundary integral methods for a capsule in a stokes flow, Inter. J. Numer. Meth. Engineering, 83(7), 829-850, 2010. 


\section{T. MILCENT AND E. MAITRE}

[29] Jian-Jun Xu, Zhilin Li, John Lowengrub, and Hongkai Zhao, A level-set method for interfacial flows with surfactant, J. Comput. Phys., 212(2), 590-616, 2006.

[30] Yang Zhang, Ken Kamrin, and Jean-Christophe Nave, An Eulerian numerical method for fluidsolid interaction, in APS Division of Fluid Dynamics Meeting Abstracts, 1, 2009. 